\title{
A survey of Opiinae (Hymenoptera, Braconidae) of Finland, part 1
}

\author{
Maximilian Fischer \& Martti Koponen
}

Fischer, M. \& Koponen, M. 1999: A survey of Opiinae (Hymenoptera, Braconidae) of Finland, part 1. — Entomol. Fennica 10: 65-93.

Faunistic records of 150 species of Opiinae are given mainly for Finland. 78 species are reported as new to Finland. The gross distributions and known hosts are mentioned. In Finnish collections were found 9 species new to Estonia, 7 to Greece, 2 to Netherlands, 9 to Poland, 2 to Russia, 5 to Sweden and 10 to the Canary Islands. Oncophanes flaviceps Hellén is a new synonym of Opius (Aulonotus) pilosiscutum Fischer. Paraphytomyza fulvicornis (Hendel) (Diptera, Agromyzidae) for Opius (Utetes) rotundiventris Thomson and Phytomyza hendeli Hering (Diptera, Agromyzidae) for Opius (Cryptonastes) tersus (Foerster). As suggested by the editors of Entomologica Fennica this article is divided in two parts. Of the 150 examined species are handled in this part and 80 in second part. The distribution maps for all 150 species as well as the list of references are in part two.

Maximilian Fischer, Natural History Museum Vienna, 2. Zoological Department, Burgring 7, A-1014 Vienna, Austria

Martti Koponen, Department of Applied Zoology, P. O. Box 27, FIN-00014 University of Helsinki

Received 1 February 1998, accepted 2 July 1999

\section{Introduction}

The first species of Opiinae from Finland were reported by Krogerus (1927) and Hellén (1946). After that, mainly Fischer has determined new Opiinae material from the Finnish Museum of Natural History, Zoological Museum, University of Helsinki (abbreviated MZH). Results of these determinations have been published by Fischer (from 1957) and Hellén (1958, 1959). According to the literature, 75 species were known from Finland. Frequently, collecting places and names of collectors have been mentioned but practically no collecting dates or host records.

Van Achterberg \& Salvo (1997) proposed a separation of a genus Phaedrotoma Foerster from Opius Wesmael, 1835, which was used by Fischer before on the basis of the shape of the mandible. They ascribe species with a derived mandible (e.g. with expansion on the lower margin near base) to Opius Wesmael, while the rest are called Phaedrotoma Foerster, 1962.

This idea was not new. Fischer thought of this possibility decades ago, but hesitated to publish it. In 1998, at which time he was not aware of the paper of van Achterberg and Salvo, he separated Opius Wesmael s.str. from Utetes Foerster, 1862 on the basis of the basal expansion of the mandible. However, in a forthcoming paper he decided to take back this decision for the time being, for 
the following reasons: (a) more than 1000 species are described worldwide, which one would have to examine individually to see if they belong to one genus or another. The whole system for the identification of the taxa would be disturbed; (b) there are sometimes gradual transitions between the characters; (c) species which are very close to each other would be placed in different genera; and (d) the majority of the former Opius would go to Phaedrotoma Fischer can not easily become familiar with this idea, but it is, however, not a genuine argument against the separation of two genera.

The host records are an assemblage of the data given in previous papers, either by Fischer or other authors. The great majority of the data was compiled by Fischer (1972). In the latter publication, one can trace back the sources of the records given (for example, page 436: Opius [H.] latipes Fischer: Phytomyza cineracea Hendel von Ranunculus acris L. [tide Fischer 1967]; or page 131: Opius [A.] reconditor Wesmael: Rubiomyza $x y$ lostei Robineau-Desvoidy [fide Rondani 1872], etc.).

\section{Material}

For this survey about 3000 specimens (1305 ond 1692 o ) of Opiinae from Finland have been investigated. The material is deposited mainly in the Department of Applied Zoology (abbreviated DAZH, curator M. Koponen) and the Finnish Museum of Natural History, Zoological Museum (MZH, A. Albrecht), University of Helsinki. Some specimens have also been found in the collections of the Department of Biology, Turku University (TUU, S. Koponen). The greater part of the data was determined by Fischer, with a lesser amount studied by M. Koponen.

The names of collecting places (without grid numbers), collecting dates (as far as known) and names of collectors are listed for 150 species found in Finland. A distribution of species is shown on maps. For gross distributions, new records of these species from other countries are also mentioned, based on material found in Finnish collections. The host records are mainly from the literature and files of Fischer, since the reared material from Finland is scanty.

The collecting dates for older specimens are usually not shown on the labels. Instead, there are often code number labels. The explanations for these numbers are in the diaries or date books of individual collectors. For this survey, Koponen has used the diaries (deposited in the MZH) of W. Hellén and some other collectors. Some diaries or date books, e.g. of Y. Hukkinen, V. Kanervo and J. Listo (with collections from the Agricultural Research Centre, Department of Pest
Investigation) are in the DAZH. Besides collection dates and more accurate place names, these sources may also provide interesting information about habitat or host records, which are most often missing from the pinned specimens.

\section{Biology}

Opiinae are solitary koinobiont endoparasitoids of Cyclorrhapha (Diptera) larvae. They often parasitize a late larvar instar, but species are known to infest eggs and early instar larvae. The mostfavored host families are Agromyzidae, Anthomyiidae, Tephritidae and Ephydridae.

Opius (Apodesmia) fulvicollis Thomson, $O$. (A.) nitidulator (Nees), O. (A.) ruficeps Wesmael and $O$. (A.) rufipes Wesmael are parasitoids of the anthomyiid pests Pegomya betae (Curtis) and/or Pegomya hyoscyami (Panzer) on Beta vulgaris. These parasitoids appear most frequently during the periods of heavy infestations of beetroot cultivations. At other times, at least Opius nitidulator and $O$. rufipes seem to be rare. From 1901 to 1962 in Finland there has been 20 years with more or less heavy infestations, the most serious calamities were in 1923 and 1960 (Vappula 1962). In our insect collections, there are specimens of mentioned parasitoids from that 60 -years period only from 16 years, 5 of which coincide with the years of heavy infestations. One reason for the small number of specimens in collections is casual collecting methods.

\section{Distribution}

Of the 150 species found in Finland, 66 species are found only in Europe, 61 also in Asia, 22 are Holarctic. 10 species are found also in Northern Africa and one even in South Africa.

Besides Finland, Opius (Aulonotus) pilosiscutum Fischer is known also in Algeria, Opius (Misophthora) iuxtangelum Fischer, $O$. (Nosopoea) pirchitticola Fischer and $O$. (Adontopius) adentatus Fischer also in Austria, O. (Misophthora) caprifolii Fischer also in Croatia, O. (Utetes) curtipectus Fischer also in the former Czechoslovakia, O. (Nosopoea) sigmodus Papp, O. (Odontopoea) epulatus Papp, O. (Stomosema) gyoerfii Fischer and $O$. (Opius) circinus Papp also in Hun- 
gary, O. (Hypocynodus) mongolaltaiensis Fischer also in Mongolia, O. (Opius) subpallipes Tobias also in Moldova, O. (Allophlebus) padidalis Fischer, and $O$. (Opiothorax) abditiformis Fischer also in Slovakia, O. (Agnopius) austriacus Fischer also in Austria and Hungary, O. (Nosopoea) diabolicus Fischer also in Austria and Switzerland, O. (Odontopoea) paranivens Fischer also in the Canary Islands (Tenerife) and Hungary, O. (Opius) gigapiceus Fischer also in Czechia and Hungary, O. (Opiothorax) minusculae Fischer also in England and Hungary, Opius (Opiothorax) dureseaui Fischer also in France and Hungary, O. (Kainopaeopius) tscheki Fischer also in Austria and Bulgaria, and $O$. (Opiothorax) inflammatus Fischer also in Austria, Greece and Hungary.

\section{New species to Finland}

The following 78 species are reported as new to Finland: Opius (Xynobius) decoratus Stelfox, $O$. $(X$.$) notabilis Fischer, O$. (X.) rudis Wesmael, $O$. (Aulonotus) holconotus Fischer, O. (A.) pilosiscutum Fischer, O. (Apodesmia) aethiops Haliday, $O$. (A.) karesuandensis Fischer, O. (A.) polyzonius Wesmael, O. (Opiognathus) pactus Haliday, $O$ (Utetes) aemulus Haliday, $O$. (U.) curtipectus Fischer, $O$. (U.) magnus Fischer, $O$. (U.) ochropus Thomson, O. (Nosopaeopius) aureliae Fischer, $O$. (N.) flavigaster Fischer, $O$. (Kainopaeopius) crassicrus Thomson, $O$. (K.) speciosus Fischer, O. (K.) tscheki Fischer, O. (Cryptonastes) gracilis Fischer, $O$. (C.) minor Fischer, O. (C.) pygmaeus Fischer, $O$. (C.) tersus (Foerster), $O$. (Misophthora) caprifolii Fischer, O. (M.) iuxtangelum Fischer, $O$. (Agnopius) austriacus Fischer, $O$. (A.) heringi Fischer, O. (A.) similis Szépligeti, $O$. (A.) similoides Fischer, O. (A.) tirolensis Fischer, $O$. (Allotypus) saevulus Fischer, O. (A.) saevus Haliday, $O$. (Nosopoea) celsus Haliday, $O$. (N.) maculipes Wesmael, $O$. (N.) pirchitticola Fischer, $O$. (N.) seebensteinensis Fischer, $O$. (N.) sigmodus Papp, $O$. (N.) tuberculifer Fischer, $O$. (N.) tuberculifer Fischer ssp. diabolicus Fischer, $O$. (N.) viennensis Fischer, O. (Odontopoea) connivens Thomson, O. (O.) epulatus Papp, O. (O.) paranivens Fischer, $O$. (Lissosema) parvungula Thomson, Opius (Opius) agromyzicola Fischer, Opius (Opius) breviscapus Thomson, O. (Opius) caricivorae Fischer, O. (O.) circinus Papp, O. (O.) subpallipes Tobias, $O$. (Phaedrotoma) pulchriventris Fischer, O. (Allophlebus) fuscipennis Wesmael, O. (A.) padidalis Fischer, O. (A.) pulcherrimus Fischer, $O$. (A.) staryi Fischer, $O$. (Hypocynodus) crassipes Wesmael, O. (H.) mongolaltaiensis Fischer, O. (Adontopius) adentatus Fischer, O. (Opiothorax) abditiformis Fischer, $O$. (O.) dureseaui Fischer, O. (O.) funebris Wesmael, $O$. (O.) inflammatus Fischer, O. (O.) minusculae Fischer, O. (O.) mirabilis Fischer, O. (O.) opacus Fischer, $O$. (O.) soenderupianus Fischer, $O$. (O.) turcicus Fischer, Psyttalia concolor (Szépligeti), $P$. rhagoleticola (Sachtleben), Eurytenes abnormis (Wesmael), E. campanariae Fischer, E. cubitalis Fischer, E. impatientis Fischer, $E$. vockerothi Fischer, Biosteres (Chilotrichia) punctiscuta (Thomson), B. (Biosteres) colorativentris (Fischer), B. (B.) magnicornis (Wesmael), and Desmiostoma parvulum (Wesmael).

\section{New species to other countries}

In the DAZH and MZH there was found several species new to other countries. Below are listed only those species which have been found also in Finland. Opius (Agnopius) similis Szépligeti, $O$. (Nosopoea) celsus Haliday, O. (Opius) gigapiceus Fischer, $O$. (Odontopoea) connivens Thomson, $O$. (Lissosema) parvungula Thomson, O. (Opius) breviscapus Thomson, O. (Hypocynodus) latipes Fischer, O. (Opiothorax) magnicauda Fischer, and $O$. (Pendopius) pendulus Haliday are new to Estonia. Opius (Agnopius) similis Szépligeti, $O$. (Opius) caricivorae Fischer, O. (Phaedrotoma) diversus Szépligeti, $O$. (Pendopius) pendulus Haliday, $O$. (Hypocynodus) crassipes Wesmael, $O$. (Opiothorax) inflammatus Fischer, and $O$. (O.) longicornis Thomson are new to Greece. Opius (Utetes) aemulus Haliday and $O$. (U.) rotundiventris Thomson are new to Netherlands. Opius (Utetes) caudatus Wesmael and $O$. (U.) coracinus Thomson $O$. (U.) trisulcus Thomson $O$. (U.) zelotes Marshall, O. (Kainopaeopius) crassicrus Thomson, O. (Agnopius) tirolensis Fischer, $O$. (Nosopoea) circulator (Nees), O. (Opiothorax) magnicauda Fischer, and $O$. (O.) mirabilis Fischer are new to Poland. Opius (Nosopoea) altimontanus Fischer and Biosteres (Biosteres) magnicornis 
(Wesmael) are new to Russia. Opius (Nosopoea) circulator (Nees), O. (Opius) agromyzicola Fischer, O. (Allophlebus) staryi Fischer, O. (Opiothorax) magnicauda Fischer, and $O$. (O.) spretus Haliday are new to Sweden. Opius (Apodesmia) nitidulator (Nees), O. (Nosopoea) maculipes Wesmael, $O$. (Tolbia) rudiformis Fischer, $O$. (Phaedrotoma) diversus Szépligeti, O. (Pendopius) pendulus Haliday, O. (Opiothorax) filicornis Thomson, O. (O.) levis Wesmael, O. (O.) longicomis Thomson, Eurytenes campanariae Fischer, and E. impatientis Fischer are new to the Canary Islands of Spain.

\section{Examined species}

Tribus Ademonini

Genus Ademon Haliday

Ademon Haliday, 1833: 266.

Type species: Bracon decrescens Nees.

\section{Ademon decrescens (Nees)}

Bracon decrescens Nees, 1812: 35, 우.

Rogas decrescens; Nees 1818: 307.

Ademon decrescens; Haliday 1833: 266; Hellén 1958: 15; Fischer 1959b: 253 (redescription); 1971a: 18; 1972: 91; Tobias 1986: 9.

Distribution. Austria, the former Czechoslovakia, England, Finland (map 1), Germany, Hungary, Italy, Poland, Russia, Spain, Sweden, Mongolia.

Hosts. Diptera, Ephydridae: Hydrellia argyrogenis Becker on Potamogeton perfoliatus, $\mathrm{Hy}$ drellia cochleariae Haliday on Potamogeton crispus and P. lucens, Hydrellia fascitibia Roser on Potamogeton lucens and Alisma plantago-aquatica, Hydrellia potamogeti Hering on Potamogeton sp., Hydrellia stratiotae Hering on Stratiotes aloides; Agromyzidae: Cerodontha (Icteromyza) geniculata Fallén on Eriophorum latifolium.

Material examined. 3 o from Finland (MZH, V. Vikberg), Ab: Karislojo = Karjalohja (W. Hellén); N: Esbo = Espoo (W. Hellén leg.); Ta: Janakkala, 28.9.1995 (V. Vikberg).

\section{Ademon mutuator (Nees)}

Bracon mutuator Nees ab Esenbeck, 1812: 55, $0^{*}$.

Rogas mutuator; Nees ab Esenbeck 1834: 221. Ademon mutuator; Marshall 1891: 13; Marshall 1894: 288, 우 ơ; Hellén 1958: 15; Fischer 1959b: 255 (redescription); 1971a: 19; 1972: 65; Tobias 1986: 9.

Remark. It is not sure whether A. mutuator (Nees) is an independent species or a synonym for $A$. decrescens (Nees). It is possible that the differentiating character (sculpture on metasomal tergites) is within the range of variability of one species.

Distribution. Austria, Finland (map 2), Germany, Hungary, Corsica.

Reported from Finland (MZH) by Hellén (1958), Ab: Karislojo = Karjalohja; N: Munksnäs = Helsinki, Munkkiniemi; Ta: Sysmä; and Russian Karelia: Uslanka. We have seen only the $\sigma$ specimen from Uslanka.

Tribus Opiini

Genus Opius Wesmael

Opius Wesmael, 1853: 115. Type species: Opius pallipes Wesmael (Wharton 1986: 369).

\section{Subgenus Xynobius Foerster}

Xynobius Foerster, 1862: 235. Type species: Xynobius pallipes Foerster. In the publication of 1862 the author's name, Förster, is written as Foerster.

\section{Opius (Xynobius) decoratus Stelfox}

Opius decoratus Stelfox, 1949: 307; Fischer 1971a: 60.

Opius (Xynobius) decoratus; Fischer 1972: 92 (redescription); Tobias 1986: 29.

Distribution. former Czechoslovakia, England, Finland (map 3), Hungary.

A new species to Finland.

Host. Unknown.

Material examined. $3 \sigma^{7}$ from Finland (TUU), Ab: Nauvo, 3.7.1948 (E. K. Lahtinen), Turku, 23.6.1948 (E. K. Lahtinen). 


\section{Opius (Xynobius) notabilis Fischer}

Opius notabilis Fischer, 1958a: 64, ơ; 1971a: 92. Opius (Xynobius) notabilis; Fischer 1972: 97; Tobias 1986: 29.

Distribution. Finland (map 4), Hungary, Romania.

A new species to Finland.

Host. Unknown.

Material examined. 2 ㅇ 20 from Finland (DAZH), Ab: Nauvo, Sandö, 19.7.1978 (M. Koponen); Om: Reisjärvi, 11.7.1980 (M. Koponen).

\section{Opius (Xynobius) rudis Wesmael}

Opius rudis Wesmael, 1835: 141, 웅; Fischer 1958: 58 (key), 67 (redescription); Fischer 1971a: 106.

Opius (Nosopoeus) rudis; Thomson 1895: 2190. Opius (Xynobius) rudis; Fischer 1972: 88 (key), 98 (redescription); 1981b: 2 (distribution); Papp 1981c: 141; Tobias 1986: 26.

Neopius carinaticeps Gahan, 1917: 204.

Distribution. Austria, Belgium, former Czechoslovakia, England, Finland (map 5), France, Germany, Hungary, Ireland, Algeria, Marocco, North Korea, southern Canada, northern U.S.A.

New to Finland.

Hosts. Diptera, Agromyzidae: Agromyza nigripes Meigen on Glyceria fluitans and Holcus lanatus, Agromyza megalopsis Hering, Agromyza sp. on Hordeum sp.

Material examined. 1 from Finland (MZH), Sa: Imatra, 8.-11.6.1956 (Håkan Lindberg).

\section{Subgenus Aulonotus Ashmead}

Holconotus Foerster, 1862: 259. Type species: Opius comatus Wesamel.

Aulonotus Ashmead, 1900: 368 (nom. nov. pro Holconotus Foerster).

\section{Opius (Aulonotus) comatus Wesmael}

Opius comatus Wesmael, 1935: 145; Fischer 1958g: 249; Hellén 1959: 21; Fischer 1967b: 141; 1971a: 55; 1974: 82; Wharton 1987: 65.

Holconotus comatus; Foerster 1862: 259. Opius (Aulonotus) comatus; Fischer 1972: 105; Papp 1979a: 75.
Opius (Opius) comatus; Jakimavicius 1973: 33 (distribution).

Opius (Xynobius) comatus; Tobias 1986: 31.

Dapsilarhtra sulcifera Papp, 1967: 209, 9 (distribution). Synonymised by Fischer 1973 (1974): 48.

Opius (Xynobius) flaviceps auct.; Koponen \& Tobias 1989: 24.

Distribution. Austria, Belgium, Denmark, England, Finland (map 6), France, Germany, Hungary, Ireland, Italy, Lituania, Poland, Sweden, Israel, Mongolia, Japan, U.S.A. (Md., N.Y., S.C.).

Reported by Hellén (1957) from Finland: EH (Hauho), PH (Keuruu) and from Russia: Leningad region (Sakkola).

Host. Unknown.

Material examined. $13 \sigma^{*} 27$ f from Finland $(\mathrm{DAZH}, \mathrm{MZH}), \mathrm{Ab}$ : Nådendal = Naantali, 2.8.1937 (W. Hellén), Perniö, 20.9.1994 (M. Koponen), Nystad = Uusikaupunki, 5.9:1917 (W. Hellén); N: Esbo, Grankulla, 4.8.1915 (W. Hellén), Espoo, 10.8.1980, 29.8.1981 (M. Koponen), Helsinki, 7.-16.9.1978 (M. Koponen), 27.29.8.1982 (Y. Zhongqi), Kirkkonummi, 20.9.1975, 14.9.1980, 6.9.1989 (M. Koponen), Nurmijärvi, 5.7.1992, 13.8.-8.9.1994 (M. Koponen), Pernå = Pernaja, 14.-19.8.1945 (̊. Nordström), Sibbo, Nevas, 5.-10.8.1986 (A. Albrecht), Sipoo, 26.7.1981 (M. Koponen), Tammisaari, 13.8.1986 (M. Koponen), Tvärminne, 13.30.7.1914 (W. Hellén); Ta: Loppi, 1.8.1982, Nastola, 17.8.1980 (M. Koponen); Sa: Ristiina, 6.9.1987 (M. Koponen); Tb: Rautalampi, 14.7.1983 (M. Koponen); Oba: Kuivaniemi, 18.7.1980 (M. Koponen).

\section{Opius (Aulonotus) holconotus Fischer}

Opius holconotus Fischer, 1958g: 250, ơ; 1971a: 72.

Opius (Aulonotus) holconotus; Fischer 1972: 103 (key), 108 (redescription).

Opius (Xynobius) holconotus; Tobias 1986: 31.

Distribution. England, Finland (map 7), Hungary, Russia (Ural).

A new species to Finland.

Host. Unknown.

Material examined. $5 \sigma^{\circ}$ from Finland (DAZH, MZH), Obb: Simo, 17.7.1980 (M. Koponen); Li: Inari, Lemmenjoki, 4.7.1937 (W. Hellén leg.). 


\section{Opius (Aulonotus) pilosiscutum Fischer}

Opius pilosiscutum Fischer, 1962c: 96, ; 1971a: 100.

Opius (Aulonotus) pilosiscutum; Fischer 1972: 103 (key), 111 (redescription).

Opius (Xynobius) pilosiscutum; Tobias 1986: 31. Opius (Xynobius) comatus auct.; Koponen \& Tobias 1989: 24.

Oncophanes flaviceps Hellén, 1957: 44. New synonym. Praeoccupied by Opius flaviceps Gahan, 1915.

Distribution. Algeria, Finland (map 8).

Host. Unknown.

Material examined. 2 ㅇ from Finland (DAZH, MZH), Ta: Hauho, 24.8.1941 (W. Hellén); Obb: Kemi, 12.8.1982 (M. Koponen).

\section{Subgenus Apodesmia Foerster}

Apodesmia Foerster, 1862: 259. Type species: Apodesmia taeniata Foerster.

Therobolus Foerster, 1862: 260. Type species: Opius ruficeps Wesmael.

\section{Opius (Apodesmia) aethiops Haliday}

Opius aethiops Haliday, 183\%: 21'2, \%; Marshall 1891 :39, o*; Fischer 1958g: 255; Hellén 1959: 21; Fischer 1971a: 40.

Opius (Apodesmia) aethiops; Fischer 1972: 118 (redescription), 1984a: 93 (key).

Opius (Xynobius) aethiops; Tobias 1986: 31, 34, 37 (key).

Distribution. Austria, former Czechoslovakia, Denmark, England, Estonia, Finland (map 9), France, Germany, Hungary, Ireland, Italy, Lituania, Poland, Russia, Spain, Israel.

A new species to Estonia.

Hosts. Agromyzidae: Cerodontha denticornis Panzer or Phytomyza milii Kaltenbach.

Material examined. $123 \sigma^{\circ}$ and 769 from Finland (DAZH, MZH), Al: Hammarland, 29.6.1983 (M. Koponen), Jomala, Kungsö, 9.6.1939 (W. Hellén); Ab: Lohjan kunta, 16.5.1982, Nummi, 18.5.1979, Perniö, 3.5.1983, Turku, 19.5.1979 (M. Koponen), Nystad = Uusikaupunki, 26.7.1917 (M. Hellén), Vihti, 20.5.1984, Västanfjärd, 3.5.1983 (M. Koponen); N: Elimäki, 7.5.1982 (M. Koponen), Esbo, Hagalund, 24.5.1920 (W. Hellén),
Espoo, 30.5.1979, 11.5.1982, 5.5.1983, Hanko, 19.5.1984, 20.5.1989 (M. Koponen), Helsinge (W. Hellén), Helsinki, 26.5.1975, 23.5.1979, 6.6.1980, 5.5.1982, 15.-18.5., 16.6.1983, 25.5.1984 (M. Koponen), 22.5.1982 (Y. Zhongqi), Hoplax = Huopalahti (E. Lindqvist), Hyvinkää, 2.6.1979, 26.5.1982, 14.5.1983, 23.5.1987, Nurmijärvi, 10.5.1964, 18.5.1980, 30.5.-1.6.1982, 7.21.5.1983, 13.-24.5.1984, 29.4.-3.5.1986, 17.5., 18.6.1987, 29.5.1988, 7.5.1989, 29.4.1990, 14.31.5.1992, 7.5., 7.6.1994 (M. Koponen), Fredriksberg = Helsinki, Pasila (R. Frey), Pernaja, 9.5.1982 (M. Koponen), Porvoon mlk. 25.5.1982 (Y. Zhongqi), 3.6.1982 (M. Koponen), Sipoo, 24.5.1.6.1983, Siuntio, 30.5.1979 (M. Koponen), Tikkurila, 25.5.1920 (J. Listo), Tuusula, 8.6.1975, 15.5.1977 (M. Koponen), Täcktom = Hanko, 9.6.1955 (V. Nyström), Vantaa, 20.5.1974 (M. Koponen); St: Eura, 16.6.1982, Mouhijärvi, 29.6.1993, Säkylä, 13.6.1982, Yläne, 19.6.1982 (M. Koponen); 'la: Hartola, 11.6.1987, Hattula, 28.5.1988, Hollola, 18.5.1991, Hämeenlinna, 7.6.1980, 22.5.1982, Janakkala, 26.5.1982, 28.5.1983, Juupajoki, 3.6.1987 (M. Koponen), 13.6.1982 (Y. Zhongqi), Lammi, 7.6.1980, Pälkäne, 23.5.1982, Tammela, 30.5.1993 (M. Koponen); Sa: Hirvensalmi, 5.6.1982 (M. Koponen), Joutseno, 25.6.1940, 30.5.1958 (E. Thuneberg), Lappeenranta, 26.6.1990, Mikkelin mlk., 17.5.1974, 21.5.1977, 1.6.1980, 8.-9.5.1982, 6.5.1990, Mäntyharju, 4.6.1982, Ristiina, 27.5.1979, 4.5.-1.6.1980, 8.5., 5.6.1982, 4.6.1983, 3.7.1987, 12.5.1989, 11.5.1991, 8.4., 8.5.1993 (M. Koponen); Kl: Parikkala, 7.7.1940 (W. Hellén); Tb: Vesanto, 28.6.1991 (M. Koponen); Sb: Leppävirta, 30.5.1865 (J. A. Palmén), 16.6.1988 (M. Koponen); Kb: Liperi, 24.6.1982 (M. Koponen); Ok: Paltamo, 4.-5.7.1991 (M. Koponen); Oba: Oulu (R. Frey), 29.5.1908 (Wuorentaus), 27.6.1982 (M. Koponen), Obb: Kemi, 6.7.1982 (M: Koponen); and $110^{*} 69$ from Estonia (DAZH).

\section{Opius (Apodesmia) fulvicollis Thomson}

Opius fulvicollis Thomson, 1895: 2181, 우 o ; Hellén 1946: 132; Fischer 1958g: 256; 1971a: 67. Opius (Apodesmia) fulvicollis; Fischer 1972: 117 (key), 121 (redescription); 1984a: 92 (key). Opius (Xynobius) fulvicollis; Tobias 1986: 34. 
Opius cupidus Gahan, 1919: 162 (\%). Synonymised by Fischer 1964a: 237.

Distribution. Belgium, Finland (map 10), Germany, Netherlands, Russia (Leningrad region), Sweden, Canada, U.S.A.

Hosts. Anthomyiidae: Pegomya betae (Curtis) and $P$. hyoscyami (Panzer) on Beta vulgaris. In Finland, reared from Pegomya hyoscyami. One example of rearings took place at the Agricultural Research Centre in Tikkurila. Leaves of sugar beet infested with $P$. hyoscyami larvae were taken from a field in Vihti, Selki, Södergård, 15.7.1929 by J. Listo. The samples were put in rearing pots with sand on the bottom. By 23.7.1929 all larvae had pupated, most of them in the sand, some in the rotting leaves. The rearing pots were placed in a cellar in winter time (16.12.1929-28.4.1930). About 850 flies and 20 wasps emerged. The first few wasps emerged at the beginning of September, but most after winter (6.6.-12.7.1930).

Material examined. $43 \sigma^{*} 42 \%$ from Finland (DAZH, MZH, TUU), Al: Eckerö. 12.7.1935 (A. Saarinen); Ab: Korppoo, 5.7.1948 (E. K. Lahtinen), Turku, 12.7.1935 (A. Saarinen), Nystad = Uusikaupunki, 23.6.1918 (M. Hellén), 7.7.1918, 6.8.1923 (W. Hellén), Vihti, 1930, reared (J. Listo); N: Hangö = Hanko (Berg, Wuorentaus), 1931, 4.7.1972 (W. Hellén), Täktom = Hanko, 12.8.1903 (U. Sahlberg), Helsinge, 19.7.1915 (W. Hellén), $\mathrm{H}$ :fors = Helsinki (R. Frey, R. Tuomikoski), Kirkkonummi, 24.7.1991 (M. Koponen), Tvärminne (Håkan Lindberg), 14.7.1932 (A. Nordman), Vantaa, 22.8., 5.9.1978 (M. Koponen); Ka: Kymi, 1930, reared (J. Listo); Ta: Loppi, 1927, reared (Y. Hukkinen, V. Kanervo), Tampere (Linnaniemi); and $3 \sigma^{*}, 1$ f from Russia (MZH), Leningrad region.

\section{Opius (Apodesmia) karesuandensis Fischer}

Opius karesuandensis Fischer, 1964c: 237 (key), 우 đo: 1971a: 79.

Opius (Apodesmia) karesuandensis; Fischer 1972: 117 (key), 123 (redescription); 1984a: 92 (key); Zaykov \& Fischer 1983: 48.

Opius (Xynobius) karesuandensis; Tobias 1986: 31.

Distribution. Austria, Finland (map 11), Germany, Italy, Sweden, Canada (Yukon), U.S.A. (Alaska).
A new species to Finland.

Host. Unknown.

Material examined. 2 \% from Finland (DAZH, MZH), Ok: Paltamo (7142:554), 30.6.1991 (M. Koponen); Lkoc: Muonio, Pallasjärvi, 19.6.1953 (Wegelius leg.).

\section{Opius (Apodesmia) nitidulator (Nees)}

Bracon nitidulator Nees, 1834: $560^{\star}$.

Opius nitidulator; Haliday 1837: 213 ; Marshall 1891: 38, 우후 Hellén 1946: 133; Fischer 1958a: 43; 1958g: 261 (redescription); 1971a: 91.

Opius (Apodesmia) nitidulator; Fischer 1972: 118 (key), 135 (redescription); 1984a: 92 (key).

Opius (Xynobius) nitidulator; Tobias 1986: 34.

Opius vittatus Ruschka, 1915: 295, i ơ. Synonymised by Fischer 1958g: 261.

Distribution. Austria, Belgium, the Canary Islands (Tenerife), Czechia, England, Finland (map 12), France, Germany, Hungary, Russia, Sweden, Tunisia, Canada (Alta., B.C., Ont., Sask.), U.S.A. (Cal., Colo., Wyo.).

A new species to the Canary Islands.

Hosts. Diptera, Anthomyiidae: Pegomya betae (Curtis) on Beta vulgaris, Pegomya esuriensgroup, Pegomya hyoscyami (Panzer) on Beta vulgaris and Atriplex hortensisabove Iresine lindeni, Pegomya nigritarsis (Zetterstedt), Pegomya setaria (Meigen). Calliphoridae: Calliphora vomitoria (Linnaeus), Lucilia caesar (Linnaeus). Muscidae: Musca domestica Linnaeus. Tachinidae: Tachina sp. We expect that some records are incorrect, either by misidentification of the parasitoids (for example, by confusion with similar Alysiinae), or by misobservation from mixed rearings. Material examined: 8 o $o$ from Finland (DAZH, MZH, TUU), Ab: Korppoo, 5.7.1948, Turku, 20.-22.6.1948 (E. K. Lahtinen); N: Hangö = Hanko, 12.7.1967, 9.7.1970 (W. Hellén), Helsinki, 31.7., 13.9.1978 with pitfall traps in a sugar beet field (A.-L. Varis), Tvärminne (E. Lindqvist); Kl: Parikkala, 13.6.1945 (W. Hellén); and $30^{*}$ the Canary Islands, Tenerife (DAZH), 5.-7.4. 1993 (M. Koponen leg.).

\section{Opius (Apodesmia) ocellatus Wesmael}

Opius ocellatus Wesmael, 1835: $1460^{\pi}$; Marshall 1894: 336 우 $\sigma^{\star}$; Fischer 1958g: 263 (redescrip- 
tion); 1971a: 93; Hellén 1959: 22.

Opius (Apodesmia) ocellatus; Fischer 1972: 117 (key), 126 (redescription), 1981a: 229; 1984a: 92. Opius (Xynobius) ocellatus; Tobias 1977: 422; 1986: 31.

Opius (Rhabdospilus) areolaris Thomson, 1895: 2199, 우 $d^{7}$. Synonymised by Fischer 1958g: 263. Opius hungaricus Szépligeti, 1896: 317, 383, ㅇ. . Synonymised by Fischer 1958g: 263.

Opius (Utetes) brutus Papp, 1978b: 363, ㅇ. Synonymised by Fischer 1981a: 229.

Distribution. Austria, Belgium, England, Finland (map 13), Germany, Hungary, Italy, Sweden.

Host. Tephritidae: Philophylla heraclei (Linnaeus) on Pastinaca sativa and Heracleum sphondylium.

Material examined. $20^{*} 4$ 우 from Finland (DAZH, MZH), Al: Finström, 7.7.1984 (M. Koponen), Föglö, 26.7.1936 (W. Hellén), Sund, 1.7.1983 (M. Koponen); N: Esbo = Espoo (R. Frey), Nurmijärvi, 5.8.1990 (M. Koponen); Kb: Liperi, 4.7.1994 (M. Koponen leg.).

\section{Opius (Apodesmia) polyzonius Wesmael}

Opius polyzonius Wesmael, 1835: 136, o ; Marshall 1895: 320, ㅇ ơ; Fischer 1958g: 266; 1971a: 100.

Opius (Apodesmia) polyzonius; Fischer 1972: 118 (key), 128 (redescription); 1984a: 93.

Opius (Nosopoeus) polyzonius; Thomson 1895: 2191, ․ .

Nosopaeus (Opius) polyzonius; Fulmek 1962: 29 , $40,49$.

Opius (Xynobius) polyzonius; Tobias 1986: 36.

Distribution. Austria, Belgium, Czechia, Denmark, England, Finland (map 14), Germany, Ita1y, Poland, European Russia, Spain, Sweden, Canada (B.C., Ont., Sask.), U.S.A. (Md., Minn., N.D., N.Y.).

\section{A new species to Finland.}

Hosts. Agromyzidae: Agromyza albitarsis Meigen on Alnus rotundifolia; Agromyza alnibetulae Hendel, Agromyza baetica Griffiths on Phragmites australis; Agromyza hendeli Griffiths on Phragmites australis; Agromyza lucida Hendel, Agromyza phragmitidis Hendel on Phragmites australis; Agromyza reptans Fallén, Agromyza sp. on Elymus repens and Phragmites australis; Agromyza spenceri Griffiths, Liriomyza strigata Mei- gen, Trilobomyza labiatarum Hendel on Stachys officinalis.

Material examined. $100^{*} 12$ f from Finland (DAZH, MZH), Ab: Lojo = Lohja (Storå leg.); N: Espoo, 26.7.1983 (M. Koponen), Hangö = Hanko, 14.7.1977 (W. Hellén), 15.-20.8.1990 (A. Albrecht), Kirkkonummi, 23.5.1991 (M. Koponen), Nurmijärvi, 21.8.1976, 28.8.1982 (M. Koponen), Lappvik = Tammisaari, Lappohja (L. von Essen), Tuusula, 5.8.1975 (M. Koponen); Ta: Hattula, 1.8.1992 (M. Koponen), Jämsä, 27.8.1991 (M. Koponen); Sa: Mikkelin mlk., 23.6.1983 (M. Koponen); Tb: Keuruu, 18.-19.8.1928 (W. Hellén).

\section{Opius (Apodesmia) reconditor Wesmael}

Opius reconditor Wesmael, 1835: 134, ㅇ ㅇ ; Haliday 1837: 213, o o ${ }^{\star}$; Fischer 1971a: 104.

Opius (Apodesmia) reconditor; Fischer 1972: 118 (key), 130 (redescription); 1984a: 93 (key).

Opius (Xynobius) reconditor; Tobias 1986: 34 (key).

Distribution. Belgium, Denmark, England, Finland (map 15), Greece, Hungary, Italy, Poland.

A new species to Finland and Greece.

Hosts. Agromyzidae: Paraphytomyza luteoscutellata (de Meijere) = Phytagromyza xylostei auct., Trilobomyza labiatarum Hendel, Trilobomyza verbasci Bouché. The parasite is probably misidentified in every case.

Material examined. 10 from Finland (MZH), Oa: Lappfjärd (691:21), 16.7.1959 (G. Nordström leg.), and $10^{\pi}$ from Greece (DAZH), Corfu: Ag. Spiridon, 26.4.1994 (M. Koponen leg.)

\section{Opius (Apodesmia) ruficeps Wesmael}

Opius ruficeps Wesmael, 1935: 143, o; Fischer 1958g: 269; 1971a: 107.

Therobolus ruficeps; Foerster 1862: 260; Krogerus 1927: 47.

Opius (Apodesmia) ruficeps; Fischer 1972: 117 (key), 132 (redescription); 1984a: 92; Papp 1981c: 141.

Opius (Xynobius) ruficeps; Tobias 1986: 34.

Opius rufipes; Hellén 1946: 133, misidentified (corrected by Fischer 1958g).

Distribution. Austria, Belgium, former Czechoslovakia, Denmark, England, Finland (map 16), 
France, Germany, Hungary, Italy, Russia (Leningrad region), Sweden, former Yugoslavia, North Korea.

Hosts. Anthomyiidae: Pegomya betae (Curtis) and P. hyoscyami (Panzer) on Beta vulgaris, Pegomya terebrans $($ Rondani $)=$ nigricornis Strobl on Cirsium sp. and Carduus sp., Pegomya nigritarsis (Zetterstedt). Unknown host on Chenopodium sp. and Datura stramonium. In Finland, reared from Pegomya hyoscyami by J. Listo.

Material examined. $8 \sigma^{\circ} 12$ 우 from Finland $(\mathrm{DAZH}, \mathrm{MZH}), \mathrm{Ab}:$ Nystad = Uusikaupunki, 11.7.1918 (W. Hellén), Vihti, Selki, 1929, 1930 (J. Listo); N: Esbo = Espoo, 10.-17.7.1910 (W. Hellén), Hangö = Hanko, 12.7.1914 (W. Hellén), Helsinge $=$ Helsinki rural municipality (W. Hellén), Lappvik = Tammisaari, Lappohja (L. von Essen), Tikkurila, 1930 (J. Listo), Tvärminne (Håkan Lindberg); Ta: Pälkäne, Ristikari, 23.6.1921 (M. Hellén); Sa: Joutseno, Muukkulansaari (W. Hellén); Tb: Keuruu, 1928 (W. Hellén); Kb: Ilomantsi, 21.7.1865 (E. Grönvik leg.), and $10^{\star}, 2$ from Russia (MZH), Leningrad region.

\section{Opius (Apodesmia) rufipes Wesmael}

Opius rufipes Wesmael, 1835: 147, o*; Marshall 1891: 48, 우 $\sigma^{*}$; Fischer 1958g: 272 (redescription); 1971a: 107.

Opius (Therobolus) rufipes; Thomson 1895: 2183, 우잉.

Opius (Apodesmia) rufipes; Fischer 1972: 117 (key), 134 (redescription); 1984a: 92 (key).

Opius (Xynobius) rufipes; Tobias 1977: 422 (key); 1986: 34.

Distribution. Austria, Belgium, former Czechoslovakia, England, Finland (map 17), France, Germany, Hungary, Ireland, Italy, Norway, Poland, Russia (Lenigrad region).

A new species to Finland.

Hosts. Agromyzidae: Agromyza mobilis Meigen, Agromyza rufipes Meigen. Anthomyiidae: Pseudonupedia abbreviata (Pokorny), Pegomya acetosae Robineau-Desvoidy, Pegomya hyoscyami (Panzer) on Beta vulgaris; Pegomya nigritarsis (Zetterstedt), Pegomya setaria (Meigen) on Fallopia convolvulus. Lonchaeidae: Lonchaea nigra Meigen. Tephritidae: Philophylla heraclei Linnaeus. Diptera gen. sp. on Polygonum "von
Mangold".

Material examined. 10 \% 4 from Finland (DAZH, MZH), Al: Eckerö, Öjen, 20.8.1939 (W. Hellén); N: Helsinki, 2.8.1979 (M. Koponen), Tikkurila, 27.6.1920 (Y. Hukkinen), 10.15.7.1923 (J. Listo); Sa: Ristiina, 8.7.1979 (M. Koponen leg.), and 1 o from Russia (MZH), Leningrad region; Lavansaari, Suisaari, 8.7.1932 (W. Hellén).

\section{Subgenus Opiognathus Fischer}

Opiognathus Fischer, 1972: 140. Type species: Opius pactus Haliday.

\section{Opius (Opiognathus) pactus Haliday}

Opius pactus Haliday, 1837: 212, 우 1971a: 94.

Opius (Opiognathus) pactus; Fischer 1972: 141 (key), 142 (redesrcription); 1986: 614 (key).

?Opius (Therobolus) pactus; Thomson 1895:2182, 우 $0^{*}$.

Opius (Xynobius) pactus; Tobias 1986: 36 (key). Phaedrotoma pacta; van Achterberg 1997: 63 (new combination, not applied here).

Distribution. Austria, Czechia, Denmark, England, Estonia, Finland (map 18), Germany, Hungary, Slovakia, Switzerland.

A new species to Finland and Estonia.

Host. Unknown.

Material examined. 10 from Finland (DAZH), N: Nurmijärvi, 22.6.1975 (M. Koponen leg.), and $1 \sigma^{\star}$ from Estonia (DAZH), Pärnumaa: Massiaru, 4.6.1990 (M.Koponen leg.).

\section{Opius (Opiognathus) propodealis Fischer}

Opius propodealis Fischer, 1958c: 299, 웅 1971a: 101; Hellén 1959: 22; Papp 1979b: 355. Opius (Opiognathus) propodealis; Fischer 1972: 141 (key), 144 (redescription); 1986: 614 (key); Papp 1980b: 207.

Opius (Xynobius) propodealis; Tobias 1986: 36 (key).

Distribution. Austria, England, Estonia, Finland (map 19), France, Germany, Italy, Poland, Russia, Sweden, former Yugoslavia, North Korea.

Remark. The dorsal groove of the mesoscutum is very small. Since this character varies to a 
certain degree in the same way as others, it may more or less disappear in some minor variants. However, in most cases the sculpture on the propodeum may be sufficient to recognise the species.

Hosts. Agromyzidae: Agromyza albitarsis Meigen on Populus nigra, Agromyza frontella Rondani, Agromyza nigrella Rondani or A. mobilis Meigen on Carduus crispus L.; Phytomyza sedicola Hering on Sedum telephium ssp. maximum; Phytomyza senecionis Kaltenbach on Senecio nemorensis; Liriomyza approximata(Hendel) on Daphne mezereum; Paraphytomyza luteoscutellata $($ de Meijere) $=$ Rubiomyza xylostei (auct.) on Lonicera sp. (orientalis = ambigous!).

Material examined. $80^{*} 3$ o from Finland $(\mathrm{DAZH}, \mathrm{MZH}), \mathrm{N}$ : Kyrkslätt = Kirkkonummi $(\mathrm{R}$. Frey), Sipoo, 26.7.1981 (M. Koponen); St: Huittinen, 29.5.1993, Rauman mlk., 2.7.1986 (M. Koponen); Ta: Lammi, 12.7.1975, 23.8.1981 (M. Koponen); Sa: Mikkelin mlk., 5.6.1983, Ristiina, 4.7.1987, Rautjärvi, 29.6.1990 (M. Koponen); 1 đ $2 \%$ from Estonia (DAZH), Saaremaa: Asuka, 2.6.1990, Mõntu park, 31.5.1990 (M. Koponen); and $10^{*}$ from Poland (DAZH), Bialowieza, 8.7.1988 (M. Koponen).

\section{Subgenus Utetes Foerster}

Utetes Foerster, 1862: 261. Type species: Opius testaceus Wesmael.

According to Wharton (1987) Utetes is a genus. We prefer to retain it as a subgenus.

\section{Opius (Utetes) aemulusHaliday}

Opius aemulus Haliday, 1837: 212, + ; Fischer 1958g: 276, 278 (redescription); Fischer 1962a: 45 (ơ new); 1967b: 139; 1971a: 40.

Opius (Utetes) aemulus; Fischer 1972: 174 (key), 149 (redescription); 1981a: 227; Papp 1985: 206. Opius (Xynobius) aemulus; Tobias 1986: 39 (key). Opius melbus Papp, 1978: 367, ㅇ. Synonymised by Fischer 1981a: 227.

Phaedrotoma aethiops; van Achterberg 1997: 13.

Distribution. Austria, England, Finland (map 20), Germany, Hungary, Ireland, Italy, Netherlands, Spain.

A new species to Finland and Netherlands.

Hosts. Diptera, Agromyzidae: Liriomyza con- gesta Becker on Vicia sepium, Phytomyza ranunculi (Schrank) ssp. stolonigena Hering on Ranunculus repens.

Material examined. $90^{\circ} 21 \%$ from Finland (DAZH, MZH), N: Helsinki, 26.8.1975, 28.6., 19.8.1976, 18.9.1978, 15.9.1980, Nurmijärvi, 28.6., 23.8.1980, 27.8.1983, 22.6., 19.8., 5.9.1989, 5.8.1990, 18.6.1991, 24.6.1992, Sipoo, 24.5.1983, 26.9.1985 (M. Koponen); Ta: Janakkala, 26.5.1984, Lammi, 9.7.1975, 4.-12.7.1976, Nastola, 25.6.1994 (M. Koponen); Sa: Mikkelin mlk., 5.7.1980, Ristiina, 4.7.-16.8.1980 (M. Koponen leg.); Kb: Koli, Saivaara, 13.7.1962 (J. Grönvall leg.). Also $1 \sigma^{*}$ and 1 t from Netherlands (MZH), dunes west of Leiden and Niewekoop, 15.-25.8.1951 (Lindberg leg.).

\section{Opius (Utetes) caudatus Wesmael}

Opius caudatus Wesmael, 1835: 142, \&; Hellén 1946: 133; Fischer 1958g: 280; 1964c: 245; 1971a: 52.

Opius (Therobolus) caudatus; Thomson 1895: 2184.

Opius (Utetes) caudatus; Fischer 1972: 148 (key), 150 (redescription); 1984a: 91 (key).

Opius (Xynobius) caudatus; Tobias 1986: 43.

Opius (Therobolus) exsertus Thomson, 1895: 2184, 우 $\sigma^{\star}$. Synonymised by Fischer 1971a: 52 .

Opius exsertus; Marshall 1898: 308, 9 ơ.

Opius (Utetes) exsertus; Fischer 1972: 148 (key), 155 (redescription).

Distribution. Austria, Belgium, former Czechoslovakia, England, Finland (map 21), Germany, Hungary, Italy, Poland, western and central Russia, Sweden, Switzerland, former Yugoslavia, Japan.

A new species to Poland.

Host. Anthomyiidae: Pegomya holosteae (Hering).

Material examined. 17 o 16 우 from Finland (DAZH, MZH), Al: Hammarland, 7.7.1984 (M. Koponen), Jomala (W. Hellén), Lemland, Granholmen, 10.7.1939 (W. Hellén), Sund, 4.7.1984 (M. Koponen); Ab: Villnäs = Askainen, Hannula, 23.8.1936, Houtskär, Viken, 6.6.1936, Nystad = Uusikaupunki, 1924 (W. Hellén); N: Hangö = Hanko, 14.7., 15.8.1981 (Kock \& Perttula), 4.12.7.1989 (A. Albrecht), Nurmijärvi, 26.7.1975, 5.8.1979, 28.6.1980, 19.6.1983, 14.7.1991 (M. 
Koponen), Pernå = Pernaja, 19.8.1948 (Å. Nordström), Tvärminne, 14.-20.8.1972 (Karvonen); St: Reposaari, 24.7.1950 (V. Lauro), Suoniemi, 14.8.1945 (V. Saarinen); Ta: Hattula (L. von Essen), 11.6.1921 (M. Hellén), Hauho, 24.8.1941 (W. Hellén), Lammi, 13.7.1976, Loppi, 12.7.1991 (M. Koponen); Sa: Suomenniemi, 23.7.1978 (M. Koponen); Kl: Parikkala, 16.7.1940 (W. Hellén); Tb: Keuruu, 4.8.1928 (W. Hellén); Kb: Ilomantsi, 14.7.1865 (E. Grönvik), and 19 from Poland (DAZH), Bialowieza, 9.7.1988 (M. Koponen leg.).

\section{Opius (Utetes) coracinus Thomson}

Opius coracinus Thomson, 1895: 2185, 우 ${ }^{*}$; Hellén 1946: 133; Fischer 1958g: 284; 1971a: 57. Opius (Utetes) coracinus; Fischer 1972: 148 (key), 151 (redescription); 1984a: 91 (key).

Opius (Xynobius) coracinus; Tobias 1986: 43.

Opius silvicola Szépligeti, 1896: 317, 383, ه. Synonymised by Hischer 1958g: 284.

Distribution. Austria, former Czechoslovakia, Denmark, Finland (map 22), France, Hungary, Poland, Russia (Leningrad region), Sweden, former Yugoslavia, Turkey.

A new species to Poland.

Host. Unknown.

Material examined. $11 \sigma^{\top} 14$ 우 from Finland (DAZH, MZH), Al: Brändö, 1921, Eckerö (W. Hellén), Geta, 5.7.1984 (M. Koponen), Lemland, Flaka, 14.6.1954 (Wegelius); Ab: Houtskär, 12.7.1936 (W. Hellén), Nauvo, Seili, 18.7.1978 (M. Koponen), Finby = Särkisalo, Nystad $=$ Uusikaupunki, 13.7.1918 (W. Hellén); N: Hangö = Hanko, 20.7.1969, 11.7.1972, Helsinge $=$ Helsinki rural municipality, 1915 (W. Hellén), Helsinki, 4.8.1976 (M. Koponen), Tvärminne, 13.30.7.1914 (W. Hellén); Ta: Hämeenlinna, 20.7.1964 (M. Koponen); Sa: Mikkelin mlk., 15.8.1976, 25.6.1988, Ristiina, 23.6.1979, 23.6.1984, 23.7.1994 (M. Koponen); Kl: Parikkala, 9.-15.7.1940, 19.7.1942 (W. Hellén); 2 ㅇ from Russia (MZH), Leningrad region, and $1 \sigma^{\circ}$ from Poland (DAZH), Bialowieza, 7.7.1988 (M. Koponen leg.).

\section{Opius (Utetes) curtipectus Fischer}

Opius curtipectus Fischer, 1958g: 286, ơ; 1971a: 59.
Opius (Utetes) curtipectus; Fischer 1972: 147 (key), 153 (redescription).

Opius (Xynobius) curtipectus; Tobias 1986: 36 (key).

Distribution. former Czechoslovakia, Finland (map 23).

A new species to Finland.

Material examined. 1 ㅇ from Finland (DAZH), N: Nurmijärvi (6711:377), 21.8.1987 (M. Koponen leg.).

\section{Opius (Utetes) fasciatus Thomson}

Opius (Opius) fasciatus Thomson, 1895: 2179, 웅.

Opius fasciatus; Marshall 1898: 307 (redescription); Hellén 1946: 132; Fischer 1958g :290; 1971a: 65.

Opius (Utetes) fasciatus; Fischer 1972: 147 (key), 158 (redescription).

Opius (Xynobius) fasciatus; Tobias 1986: 36.

?Opius reconditor Haliday, 1837 (nec Wesmael 1835): 213, ๆ $o^{*}$.

Opius comparandus Fischer, 1959h: 19, ơ. Synonymised by Fischer 1972: 158.

Distribution. former Czechoslovakia, Denmark, England, Finland (map 24), Italy, Russia, Sweden.

Material examined. 1 i from Finland (MZH), Ab: Karislojo = Karjalohja, 8.7.1911 (W. Hellén leg.)

\section{Opius (Utetes) hilaris Fischer}

Opius hilaris Fischer, 1962a: 36, \&; 1971a: 72.

Opius (Utetes) hilaris; Fischer 1972: 147 (key), 149 (redescription); Fischer 1983: 14 (key); Papp 1978b: 367.

Opius (Xynobius) hilaris; Tobias 1986: 39 (key). Opius hostium Fischer, 1964d: 6, o o ; 1971a: 73. Opius (Utetes) hostium; Fischer 1972: 91 (key), 160 (redescription); Papp 1980b: 199. Synonymised by Fischer 1983.

By synonymising hostium with hilaris, the host records of the former species are to be added to those of the latter one.

Distribution. Austria, England, Finland (map 25), Germany, Hungary, Poland.

A new species to Finland.

Hosts. Agromyzidae: Agromyza nigripes Mei- 
gen on Glyceria maxima; Cerodontha (Dizygomyza) caricicola $($ Hering $)=$ soenderupi $($ Hering $)$ on Carex sylvatica; Cerodonta (Butomyza) caricivora (Groschke) on Carex sylvatica; Cerodontha (Poemyza) deschampsiae (Spencer) on Deschampsia caespitosa; Cerodontha (Phytagromyza) flavocingulata (Strobl) on Holcus lanatus; Cerodontha (Dizygomyza) iraeos (Robineau-Desvoidy in Goureau) on Iris pseudacorus; Cerodontha (Dizygomyza) luctuosa (Meigen) on Juncus effusus; Cerodontha luzulae (Groschke) on Luzula sylvatica; Cerodontha (Poemyza) pygmaea (Meigen) on Deschampsia caespitosa; Cerodontha silvatica (Groschke) on Luzula sylvatica; Paraphytomyza buhri (de Meijere) on Galium album = mollugo auct.; Liriomyza pusio (Meigen), Ophiomyia maura (Meigen), Phytomyza angelicae Kaltenbach, Phytomyza homogyneae Hendel, Phytomyza montana Groschke, Phytomyza ramosa Hendel, Phytomyza scolopendrii RobineauDesvoidy on Phyllitis scolopendrium, Phytomyza tussilaginis Hendel.

Material examined. $130^{*} 23$ from Finland (DAZH, MZH), Al: Eckerö, 2.7.1984, Hammarland, 29.6.1983, 1.7.1984 (M. Koponen); Ab: Lohjan mlk., 31.7.1979 (M. Koponen); N: Helsinki, 4.8.1976, 24.7.1979, 30.8.1980, 8.9.1983, Kirkkonummi, 24.7.1991, Nurmijärvi, 2.9.1979, 23.-24.8.1980, 14.7.1982, 10.6.1985, 23.6.1986, 30.6., 22.7., 17.9.1987, 23.8.1992 (M. Koponen leg.), Pernå = Pernaja, 12.8.1945 ( $\AA$. Nordström), Porvoon mlk., 3.6.1982 (M. Koponen), Sibbo, Nevas, 4.-10.8.1985 (A. Albrecht), Siuntio, 20.8.1978, 29.8.1979, Tuusula, 11.9.1981 (M. Koponen); Ta: Janakkala, 9.7.1983, Korpilahti, 20.8.1986, Loppi 29.8.1993 (M. Koponen); Sa: Mikkelin mlk., 11.7.1981, 18.7.1987, Ristiina, 14.8.1976, 5.7.1987 (M. Koponen); Kb: Kitee, 19.7.1982 (M. Koponen); Om: Alajärvi, 18.8.1982, Ristijärvi, 3.7.1991 (M. Koponen); Obb: Simo, 17.7.1980 (M. Koponen leg.).

\section{Opius (Utetes) magnus Fischer}

Opius magnus Fischer, 1958g: 292, q; 1971a: 85. Opius (Utetes) magnus; Fischer 1972: 147 (key), 166 (redescription); 1984a: 91 (key), 130 (syn.). Opius (Xynobius) magnus; Tobias 1977: 423 (key), 427; Tobias 1986: 41 (key).

Opius (Xynobius) sayanicus Tobias, 1977: 423 (key), 427. Synonymised by Fischer 1984a: 130. Opius scrutator Tobias, 1977: 423 (key). Synonymised by Fischer 1983: 130.

Distribution. Austria, Finland (map 26), Germany, Hungary, Italy, Poland, Caucasus, Kazakhstan, Crimea.

A new species to Finland.

Host. Tephritidae: Rhagoletis cerasi (Linnaeus) or Rhagoletis meigeni Loew on Berberis sp.

Material examined. 4 우 from Finland (DAZH), N: Helsinki, 21.8.1967 (Karvonen), 28.7.1980, 10.8.1980, 20.7.1981 (M. Koponen leg.)

\section{Opius (Utetes) ochropus Thomson}

Opius ochropus Thomson, 1895: 2185, 우 Fischer 1971a: 93.

Opius (Utetes) ochropus; Fischer 1972: 147 (key), 168 (redescription); 1984a: 91.

Opius (Xynobius) ochropus; Tobias $1986: 41$ (key).

Distribution. Finland (map 27), Russia?, Sweden.

A new species to Finland.

Host. Unknown.

Material examined. $1 \sigma^{\circ}$ from Finland (DAZH), N: Kirkkonummi, 23.7.1991 (M. Koponen leg.).

\section{Opius (Utetes) rotundiventris Thomson}

?Opius reconditor var. 3, Wesmael 1835: 134, ㅇ. Opius (Therobolus) rotundiventris Thomson, 1895: 2182, 우 ${ }^{*}$.

Opius rotundiventris; Hellén 1946: 133; Fischer 1958a: 45 (host); 1958g: 276 (key), 298 (redescription); 1971 a: 106.

Opius (Utetes) rotundiventris; Fischer 1972: 147 (key), 169 (redescription).

Opius (Xynobius) rotundiventris; Tobias 1986: 39 (key).

Distribution. Austria, former Czechoslovakia, Denmark, England, Finland (map 28), France, Germany, Hungary, Netherlands, Poland, Russia, Sweden; Japan.

A new species to Netherlands.

Hosts. Agromyzidae: Agromyza albitarsis Meigen on Populus balsamifera; Agromyza lyophaga Hering on Salix alba L.; Agromyza rufipes Meigen on Artemisia vulgaris L.; Phytomyza diversicornis Hendel, Phytomyza sedicola Hering 
on Sedum telephium maximum. In Finland reared from Paraphytomyza fulvicornis (Hendel) = langei (Hering) on Salix sp. by L. Tiensuu (new host).

Material examined. $11 \sigma^{*} 8$ f from Finland (DAZH, MZH), N: Degerö, 28.6.1945 (A. Saarinen), Helsinki, 13.9.1978, 26.6., 30.8.1980, 20.7.1981, 27.9.1982, Nurmijärvi, 14.9.1980, Tammisaari, 14.8.1986 (M. Koponen), Tvärminne, 24.-31.7.1990 (A. Albrecht); Ta: Hattula (L. von Essen), Hauho, 15.8.1935, 15.8.1941 (W. Hellén); Sa: Mikkeli, 24.7.1982 (M. Koponen); Kb: Tohmajärvi, 22.7.1982 (M. Koponen); 1 우 from Netherlands (MZH), dunes west of Leiden, 25.8.1951 (Lindberg leg.); and 1 \% from Russia (MZH), Karelia: Impilaks (F. W. Woldstedt).

\section{Opius (Utetes) trisulcus Thomson}

Opius (Therobolus) trisulcus Thomson, 1895: 2184, 웅.

Opius trisulcatus; Dalla Torre 1898: 66; Marshall 1898: 308 (redescription); Hellén 1946: 133; Fischer 1958g: 301 (redescription); 1971a: 118; Papp 1980: 22, 23 (key, distribution); 1981a: 129. Opius (Utetes) trisulcus; Fischer 1972: 148 (key), 174 (redescription); 1984a: 91 (key); Papp 1978a: 147, 148 (taxonomy).

Opius (Xynobius) trisulcus; Tobias 1986: 41.

Distribution. Austria, former Czechoslovakia, Denmark, Finland (map 29), France, Germany, Hungary, Italy, Lithuania, Poland, Sweden.

A new species to Poland.

Host. Agromyzidae: Phytobia sp. on Quercus robur (cited as pedunculata).

Material examined. $120^{\circ} 4 \%$ from Finland (DAZH, MZH), Al: Mariehamn, 3.7.1948 (A. Nordman); N: Helsinki rural municipality, 15.8.1968 (V. J. Karvonen), Helsinki, 14.8.1968 (W. Hellén), 26.7., 21.8.1980 (M. Koponen), Nurmijärvi, 22.6.1975, 23.8.1980, 17.-18.7.1981, 19.6.1983, 17.8.1985, Porvoon mlk., 26.8.1979 (M. Koponen); Ta: Hämeenlinna, 10.6.1921 (M. Hellén); and $1 \sigma^{*} 2$ f from Poland (DAZH), Bialowieza, 7.7.1988 (M. Koponen leg.).

\section{Opius (Utetes) truncatus Wesmael}

Opius truncatus Wesmael, 1835: 137, 우 $\sigma^{\text {; }}$; Marshall 1891: 43 (redescription); 1894:329 (redescription); Hellén 1946: 133; Fischer 1958g: 303 (redescription); 1971a: 119; Papp 1980a: 222 (key, distribution).

Opius (Therobolus) truncatus; Thomson, 1895: 2180 (redescription).

Opius (Utetes) truncatus; Fischer 1972: 148 (key), 175 (redescription), 1984a: 91; Papp 1978a: 147. Opius (Xynobius) truncatus; Tobias 1977: 423 (key); 1986: 41 (key, distribution).

Distribution. Austria, Belgium, former Czechoslovakia, Demnark, England, Finland (map 30), Germany, Poland, Russia, Sweden, Switzerland; Armenia, Mongolia.

Hosts. Tephritidae: Trypeta artemisiae Fabricius on Tanacetum parthenium and Artemisia vulgaris; Trypeta sp.

Material examined. 1 o 9 ㅇ from Finland (DAZH, MZH), N: Hangö = Hanko (W. Hellén), Nurmijärvi, 7.8., 28.8.1980, 8.7., 18.7.1981 (M. Koponen); St: Reposaari, 24.7.1950 (V. Lauro); Ta: Sääksmäki, 1930 (E. Kivirikko); Sa: Mikkelin mlk., 10.8.1987 (M. Koponen); Kl: Parikkala, 10.8.1942 (W. Hellén); Tb: Keuruu, 1928 (W. Hellén), and $2 \sigma^{\circ}, 2$ ㅇ from Russia, Leningrad region.

\section{Opius (Utetes) zelotes Marshall}

Opius zelotes Marshall, 1891: 40, ð’; 1894: 326; Szépligeti 1904: 166; Fischer 1958: 274 (redescription); 1971a: 123.

Opius (Apodesmia) zelotes; Fischer 1972: 118 (key), 139 (redescription).

Opius (Utetes) zelotes; Fischer 1982: 32.

Opius (Xynobius) zelotes; Tobias 1986: 39.

Opius exsertus; Fischer, 1958g: 289 (not Thomson, 1895); Hellén 1959: 22.

Opius insertus Fischer 1971a: 76; 1964e: 65 (host). Synonymised by Fischer 1982: 32.

Opius (Utetes) insertus; Papp 1978b: 363.

Host: Diptera, Anthomyiidae: Pegomya holosteae (Hering) (?).

Distribution. Austria, former Czechoslovakia, England, Finland (map 31), France, Germany, Poland, Sweden.

A new species to Poland.

Material examined. $90^{*} 9$ from Finland (DAZH, MZH), Al: Lemland, Flaka, 14.6.1954 (Wegelius); N: Helsinki, 20.8.1975 (M. Viitasaari), 11.7.1979, 18.7.1989, 20.7.1981 (M. Koponen), Kirkkonummi, 24.7.1991, Nurmijärvi, 17.7., 
9.8.1981, 23.8.1992, 16.7.1994 (M. Koponen); Ta: Forssa (R. Tuomikoski); Sa: Mikkelin mlk., 11.7.1981, Taipalsaari, 3.7.1975 (M. Koponen); and $20^{\circ} 1$ f from Poland (DAZH), Bialowieza, 7.7.1988 (M. Koponen leg.).

\section{Subgenus Nosopaeopius Fischer}

Nosopaeopius Fischer, 1972: 206. Type species: Opius ochrogaster Wesmael.

\section{Opius (Nosopaeopius) aureliae Fischer}

Opius aureliae Fischer, 1957c: 343, ㅇ; 1967b: 139; 1974: 82.

Opius (Nosopaeopius) aureliae; Fischer 1972: 206 (key), 211 (redescription); 1986: 609; Papp 1979a: 73.

Opius (Cryptonastes) aureliae; Tobias 1986: 49 (key).

Biosteres aureliae; Wharton 1988: 351.

Distribution. Austria, former Czechoslovakia,

Finland (map 32), Russia, U.S.A. (Washington).

A new species to Finland.

Host. Unknown.

Material examined. $2 \sigma^{*} 3$ from Finland (DAZH), Ab: Perniö , 20.9.1994; N: Helsinki, 1.8.9.1978; Sa: Mikkelin mlk., 22.8.1975 (all M. Koponen leg.).

\section{Opius (Nosopaeopius) flavigaster Fischer}

Opius flavigaster Fischer, 1957c: 348, ơ ; 1971a: 66.

Opius (Nosopaeopius) flavigaster; Fischer 1972: 206 (key), 218 (redescription); 1986: 609.

Opius (Cryptonastes) flavigaster; Tobias 1986: 45 (key).

Distribution. Finland (map 33), Hungary, Romania.

A new species to Finland.

Host. Unknown.

Material examined. $10^{*} 1$ ㅇ from Finland (MZH), Ab: Pojo, Åminne, 14.7.1965 (M. Meinander leg.).

\section{Subgenus Kainopaeopius Fischer}

Kainopaeopius Fischer, 1986: 609. Type species: Opius crassicrus Thomson.

\section{Opius (Kainopaeopius) crassicrus Thomson}

Opius crassicrus Thomson, 1895: 2193, 우; Fischer 1971a: 58.

Opius (Nosopaeopius) crassicrus; Fischer 1972: 206 (key), 214 (redescription); Papp 1982a: 243. Opius (Kainopaeopius) crassicrus; Fischer 1986: 610.

Opius (Cryptonastes) crassicrus; Tobias 1986: 50 (key).

Opius nigricinctus Fischer, 1957c: 353, ․ Synonymised by Fischer 1971a: 58.

Distribution. Austria, Bulgaria, former Czechoslovakia, Finland (map 34), Hungary, Poland, Romania, Russia (Leningrad region), Sweden.

A new species to Finland and Poland.

Host. Unknown.

Material examined. $260^{*} 23$ from Finland (DAZH), Ab: Västanfjärd, 6.8.1982 (M. Koponen); N: Espoo, 4.9.1979, 29.8.1981, 25.7.1983, Helsinki, 19.-29.8.1976, 20.7.1981, 2.-22.8.1982 (M. Koponen), 14.8.1982 (Y. Zhongqi leg.), Nurmijärvi, 23.7.1980, 30.7.1982, 27.8.1983, 31.7., 15.9.1984, 13.8.1987, Sipoo, 26.7.1981, Siuntio, 30.8.1981, Vantaa, 31.7.1975 (M. Koponen); St: Nakkila, 10.8.1992, Rauma, 1.7.1976 (M. Koponen); Sa: Mikkelin mlk., 10.7.1981, 31.7.3.8.1983, 30.7.1990, Puumala, 6.9.1981, Ristiina, 14.8.1976, 6.9.1981, 20.8.1983, 19.7.1987, Valkeala, 28.7.1977; Tb: Rautalampi, 15.19.7.1983 (M. Koponen); Sb: Pielavesi, 28.6.1989, Tervo, 18.7.1983 (M. Koponen); Kb: Liperi, 4.8.1993 (M. Koponen); Oba: Ii, 11.8.1982 (M. Koponen); and 10 from Poland (DAZH), Bialowieza, 6.7.1988 (M. Koponen leg.).

\section{Opius (Kainopaeopius) tscheki Fischer}

Opius tscheki Fischer, 1957c: 357, ơ; 1971a: 119; 1977: 363 (holotype designated).

Opius (Nosopoeaopius) tscheki; Fischer 1972: 207 (key), 226 (redescription).

Opius (Kainopaeopius) tscheki; Fischer 1986: 610.

Opius (Allotypus) tscheki; Tobias 1986: 52 (key). Distribution. Austria, Bulgaria, Finland (map 35).

A new species to Finland.

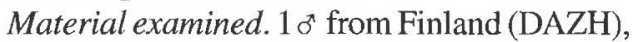
Ta: Janakkala (6754:369), 12.9.1982 (M. Kopo- 
nen leg.).

\section{Subgenus Cryptonastes Foerster}

Cryptonastes Foerster, 1862: 260. Type species: Cryptonastes tersus Foerster.

\section{Opius (Cryptonastes) curvatus Fischer}

Opius curvatus Fischer, 1957b: 213; 1964d: 3 (redescription); 1971a: 59.

Opius (Cryptonastes) curvatus; Fischer 1972: 180 (key), 187 (redescription); Papp 1982a: 243; Fischer 1984b: 37 (key); Tobias 1986; 49.

Distribution. Armenia, Austria, Estonia, Finland (map 36), Germany, Hungary, Italy, Kazakhstan, Romania, Russia, Sweden.

Hosts. Diptera, Agromyzidae: Phytomyza angelicae Kaltenbach and Phytomyza angelicastri Hering on Angelica sylvestris, Phytomyza heracleana Hering on Heracleum sphondylium, Phytomyza obscurella Fallén? on Aegopodium podagraria.

A new species to Finland and Estonia.

Material examined. $50^{*} 6 \%$ from Finland (DAZH), Al: Finström, 7.7.1984; N: Helsinki, 28.6., 20.7.1976, 12.8.1977, 11.7.1978, Nurmijärvi, 14.6., 27.8.1983; EH: Lammi, 8.7.1975; Sa: Ristiina, 4.6.1983 (all M. Koponen leg.); and $1 \sigma^{*}$ 1 o from Estonia (DAZH), Saaremaa: Kübassaare, 30.5.1990, Lihulinna, 2.6.1990 (M. Koponen leg.).

\section{Opius (Cryptonastes) gracilis Fischer}

Opius gracilis Fischer, 1957c: 337, 웅 1971a: 70.

Opius (Cryptonastes) gracilis; Fischer 1972: 179 (key), 191 (redescription); 1982: 26; 1984b: 37 (key); Papp 1981c: 152; Tobias 1986: 49 (key, distribution).

Distribution. Austria, Bulgaria, north Caucasus, former Czechoslovakia, Denmark, England, Finland (map 37), France, Hungary, Kazakhstan, Poland, Russia, Switzerland, former Yugoslavia. A new species to Finland.

Hosts. Diptera, Agromyzidae: Liriomyza sp. on Scorzonera purpurea, Liriomyza strigata (Meigen) on Pisum sativum, Phytomyza atricornis Meigen on Centaurea jacea, Phytomyza origani
Hering, Phytomyza pulsatillae Hering or Agromyza sp. on Medicago sativa, Phytomyza silai Hering on Silaum silaus. Unknown host from leaf mine on Peucedanum cervaria.

Material examined. $20^{\circ} 4$ f from Finland (DAZH), N: Nurmijärvi, 16.8.1992; Ta: Nastola, 22.6.1980; Sa: Mikkelin mlk., 29.7.1980; Kb: Tohmajärvi, 21.7.1982; Ks: Kuusamo, 4.7.1979 (all M. Koponen leg.).

\section{Opius (Cryptonastes) minor Fischer}

Opius minor Fischer, 1957b: 219; 1971a: 88.

Opius (Cryptonastes) minor; Fischer 1972: 179 (key), 197 (redescription); 1984b: 38.

Opius (Cryptonastes) gracilis Fischer var. minor; Tobias 1986: 49 (key, distribution).

Distribution. Austria, former Czechoslovakia, Denmark, England, Finland (map 38), Germany, Italy, Russia, Spain, Sweden, Switzerland, central Ural, Mongolia, Uzbekistan, Tunisia.

A new species to Finland.

Hosts. Diptera, Agromyzidae: Agromyza bicophaga Hering on Vicia tetrasperma, Agromyza genistae Hendel on Genista tinctoria, Amauromyza (Trilobomyza) gyrans (Fallén), Liriomyza congesta (Becker) on Trifolium dubium, Liriomyza sp. on Centaurea jacea, Phytomyza ferulae Hering ssp. dauci Spencer on Daucus sp., Phytomyza sphondylii Robineau-Desvoidy, Paraphytomyza luteoscutellata (de Meijere) =xylostei (auct.).

Material examined. $70^{7} 25$ from Finland (DAZH), Al: Finström, 5.7.1984; Ab: Nauvo, Seili, 17.7.1978; N: Nurmijärvi, 31.8.1979, 19.6.1983, 9.7., 15.9.1984, 27.8.1985, 1.8.1987, 9.7.1991, 25.7.1992, 20.5.1993, 17.7.1994; St: Eura, 7.7.1981, Nakkila, 10.8.1992, Rauman mlk., 2.7.1986; Ta: Janakkala, 24.8.1980, Lammi, 13.7.1976, Nastola, 17.8.1980; Sa: Mikkelin mlk., 24.7.1976, 5.7., 17.8.1980, 23.6.1983, 30.7.1990, Ristiina, 26.8.1978, 21.6.1985, 6.7., 6.9.1987; Kl: Rautjärvi, 30.6.1990; Tb: Äänekoski, 18.8.1982; Ks: Kuusamo, 3.7.1979; Lkoc: Kittilä, 8.7.1989 (all M. Koponen leg.).

\section{Opius (Cryptonastes) pygmaeus Fischer}

Opius pygmaeus Fischer, 1962c: 98, 우; 1971a: 103.

Opius (Cryptonastes) pygmaeus; Fischer 1972: 
179 (key), 198 (redescription); 1984b: 37 (key); Tobias 1986: 45 (key, distribution).

Distribution. Austria, England, Estonia, Finland (map 39), France, Hungary, Kazakhstan, Russia, northern Caucasus, Algeria.

A new species to Finland and Estonia.

Host. Diptera, Agromyzidae: Phytomyza adjuncta Hering.

Material examined. $140^{*} 26$ from Finland (DAZH), Al: Eckerö, 7.7.1984, Finström, 7.7.1984, Jomala, 9.7.1984, Saltvik, 28.6.1983 (M. Koponen); Ab: Nauvo, Seili, 17.7.1978, Perniö, 6.8.1982, Vihti, 11.7.1982 (M. Koponen); $\mathrm{N}$ : Helsinki, 10.8.1975, 25.8.1982, 25.8.1992, Nurmijärvi, 5.8.1975, 11.6.1979, 3.8.1982, 13.7.1985, 28.5.-9.6.1989, 13.8.1994 (M. Koponen); St: Rauman mIk., 2.7.1986 (M. Koponen); Ta: Hämeenlinna, 22.8.1981, Hattula, 24.5.1975 (E. Valkeila), Heinolan mlk., 5.-25.6.1983, Janakkala, 2.8.1975, 25.6.1984, 12.6.1988 (M. Koponen); Sa: Ristiina, 5.8.1978, 6.7.1987, 21.7.1989 (M. Koponen); Kl: Parikkala, 30.6.1990 (M. Koponen); Kb: Liperi, 24.6.1982, Tohmajärvi, 23.7.1982 (M. Koponen); Ok: Ristijärvi, 3.7.1991 (M. Koponen); Oba: Utajärvi, 14.8.1982 (M. Koponen); Lkor: Sodankylä, 30.7.1993 (M. Koponen); Li: Inari, 6.7.1989 (M. Koponen leg.); and $10^{*} 2$ from Estonia (DAZH), Saaremaa: Lihulinna, 2.6.1990 (M. Koponen leg.).

\section{Opius (Cryptonastes) radialis Fischer}

Opius radialis Fischer, 1957c: 341, ơ; Hellén 1959: 22; Fischer 1967a: 344; 1971a: 104.

Opius (Cryptonastes) radialis; Fischer 1972: 180 (key), 200 (redescription); 1984b: 37 (key); Papp 1979a: 88; Tobias 1986: 49 (key).

Distribution. Austria, Finland (map 40), Hungary.

Host. Unknown.

Material examined. 1 from Finland (MZH), Al: Lemland, Flaka, 12.-24.8.1954 (Håkan Lindberg leg.).

\section{Opius (Cryptonastes) tersus (Foerster)}

Cryptonastes tersus Foerster, 1862: 260.

Opius tersus; Fischer 1964c: 431; 1971a: 116.

Opius (Cryptonastes) tersus; Fischer 1972: 179

(key), 203 (redescription); Tobias 1986: 45, 58.
Opius consors Fischer, 1957c: 334, o o $\sigma^{\star}$. Synonymised by Fischer 1964c: 431.

Distribution. Austria, Estonia, Finland (map 41), Germany, Hungary, Russia, North Africa, North Korea.

A new species to Finland and Estonia.

Hosts. Diptera, Agromyzidae: Liriomyza pisivora Hering on Pisum sativum, Phytomyza scotica Hendel on Salvia pratensis, Amauromyza (Trilobomyza) flavifrons (Meigen) on Silene latifolia ssp. alba. In Finland reared from Phytomyza hendeli Hering by L.Tiensuu.

Material examined. $70^{*} 3 \%$ from Finland (DAZH, MZH), Al: Lemland, 6.7.1984 (M. Koponen); Ab: Vihti, 21.5.1989 (M. Koponen); N: Helsinki, 17.7.1951. (L. Tiensuu), Nurmijärvi, 10.7.1982, 19.6.1984, 28.5.1989 (M. Koponen); Ta: Hartola, 10.6.1987 (M. Koponen); Kl: Parikkala, 29.6.1990 (M. Koponen); Kb: Tohmajärvi, 21.7.1982 (M. Koponen), and $1 \sigma^{*}$ from Estonia (DAZH), Saaremaa: Mäebe, 31.5.1990 (M. Koponen leg.).

\section{Subgenus Misophthora Foerster}

Misophthora Foerster, 1862: 267. Type species: Misophthora laevigata Foerster.

\section{Opius (Misophthora) caprifolii Fischer}

Opius caprifolii Fischer, 1967a: 327, ㅇ․ Opius (Misophthora) caprifolii; Fischer 1972: 229 (key), 238 (redescription).

Opius (Allotypus) caprifoliae; Tobias 1986: 55 (key).

Distribution. Croatia, Finland (map 42).

A new species to Finland.

Host. Diptera, Agromyzidae: Paraphytomyza luteoscutellata $($ de Meijere $)=x y l o s t e i$ (auct.) on Lonicera caprifolium.

Material examined. 1 \% from Finland (DAZH), Ta: Hattula, 1.8.1992 (M. Koponen leg.).

\section{Opius (Misophthora) instabilis Wesmael}

Opius instabilis Wesmael, 1835: 126, \& o ; Fischer 1958d: 249; 1971a: 76.

Opius (Misophthora) instabilis; Fischer 1972: 230 (key), 247 (redescription); 1982: 27, 30.

Opius (Allotypus) instabilis; Tobias 1986: 52 
(key).

Opius fallax Szépligeti, 1896: 315, 381 \% ; Fischer 1958d: 247; 1971a: 65; Hellén 1959: 22. Synonymised by Fischer 1982: 27.

Opius (Misophthora) fallax; Fischer 1972: 228

(key), 243.

[Opius monticola Szépligeti, 1898: 392, 404, ㅇ. . Synonymy dissolved by Fischer 1982: 23 (key), 31 (redescr.).]

Distribution. Austria, Belgium, former Czechoslovakia, England, Finland (map 43), France, Germany, Hungary, Italy, Russia, Sweden, Kazakhstan (Akmolinsk), North Africa.

Remark. Since there have been synonymical alternations, it is not sure that the above-recorded distribution is correct.

Hosts. Diptera, Agromyzidae: Agromyza spiraeae Kaltenbach on Potentilla repens, Phytomyza ramosa Hendel on Knautia arvensis.

Material examined. $10^{*} 3$ from Finland (DAZH, MZH), Al: Sund, 1.7.1983 (M. Koponen); N: Elimäki, 21.6.1981, Nurmijärvi, 20.7.1975 (M. Koponen); Sa: Mikkelin mlk., 17.8.1976 (M. Koponen leg.).

\section{Opius (Misophthora) iuxtangelum Fischer}

Opius (Misophthora) iuxtangelum Fischer, 1978: 47, ㅇ․

Opius (Allotypus) iuxtangelum; Tobias 1986: 58.

Distribution. Austria, Finland (map 44).

A new species to Finland.

Host. Unknown.

Material examined. 1 f from Finland (DAZH), N: Nurmijärvi (6715:376), 23.8.1980 (M. Koponen leg.).

\section{Opius (Misophthora) pulicariae Fischer}

Opius pulicariae Fischer, 1969b: 67, 우열 1971a: 102.

Opius (Misophthora) pulicariae; Fischer 1972: 228 (key), 260 (redescription).

Opius (Allotypus) pulicariae; Tobias 1986: 55. Opius pulicariae: Perepetchayenko 1994: 37 (distribution)

Distribution. Austria, Finland (map 45), Germany, Hungary, Switzerland, Ukraine, Armenia.

A new species to Finland.

Hosts. Diptera, Agromyzidae: Ophiomyia pu- licaria (Meigen) on Picris hieracioides. and Taraxacum officinale, Phytomyza atricornis Meigen on Picris hieracioides, Phytomyza ranunculi (Schrank) on Ranunculus acris, Phytomyza virgaureae Hering.

Material examined. $2 \sigma^{*} 5$ from Finland (DAZH, MZH), N: Nurmijärvi, 25.7.1986, 20.5.1990, 9.7.1994 (M. Koponen); Ta: Lammi, 15.7.1975 (M. Koponen); Kb: Liperi, 3.7.1994 (M. Koponen); Ks: Kuusamo, 4.7.1979 (M. Koponen); Le: Enontekiö, Kilpisjärvi, 4.9.1964 (U. Erikson leg.).

\section{Subgenus Agnopius Fischer}

Agnopius Fischer, 1982: 21. Type species: Opius similis Szépligeti.

\section{Opius (Agnopius) austriacus Fischer}

Opius austriacus Fischer, 1958d: 242, ㅇ.

Opius (Misophthora) austriacus; Fischer 1972: 228 (key), 232 (redescription).

Opius (Agnopius) austriacus; Fischer 1982: 22. Opius (Allotypus) austriacus; Tobias 1986: 55.

Distribution. Austria, Finland (map 46), Hungary.

A new species to Finland.

Host. Unknown.

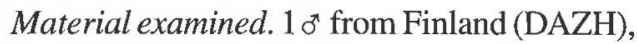
Ta: Lahti, 23.6.1988 (M. Koponen leg.).

\section{Opius (Agnopius) basirufus Fischer}

Opius basirufus Fischer, 1958d: 245, q; 1959f: 423; Hellén 1959: 21; Fischer 1967b: 139; 1971a: 46; 1974: 82.

Opius (Misophthora) basirufus; Fischer 1972: 229 (key), 234 (redescription).

Opius (Agnopius) basirufus; Fischer 1982: 22. Opius (Allotypus) basirufus; Tobias 1986: 59. Opius nodatus Fischer, 1958d: 257, 웅. Synonymised by Fischer 1971a: 46.

Distribution. Austria, Bulgaria, England, Finland (map 47), France, Hungary, Poland, Russia, Turkey.

Hosts. Diptera, Agromyzidae: Agromyza genistae Hendel, Amauromyza (Trilobomyza) gyrans (Fallén) on Campanula trachelium, Liriomyza centaureae Hering on Centaurea nigra, Liriomyza 
flaveola (Fallén) on Festuca gigantea, Phytomyza affinis Fallén on Cirsium acaulon, Phytomyza bellidina Hering on Bellis sylvestris, Phytomyza milii Kaltenbach on Holcus lanatus, Phytomyza myosotidis Nowakowski on Myosotis sylvatica, Phytomyza periclymenii de Meijere on Lonicera periclymenium L.; Phytomyza lonicerae Robineau-Desvoidy $=x y l o s t e i$ (Kaltenbach) on Symphoricarpus rivularis.

Material examined. $70^{*} 19$ from Finland (DAZH, MZH), Al: Hammarland, 29.6.1983 (M. Koponen); N: Espoo, 25.8.1979, 10.8.1980, Helsinki, 15.8.-4.9.1978, 28.8., 26.9.1979, Mäntsälä, 2.10.1987, Nurmijärvi, 2.9.1978, 16.5.-2.6.1979, 16.6.1985, 1.7.1992 (M. Koponen), Sibbo, Nevas, 8.-28.7.1985 (A. Albrecht); St: Huittinen, 29.5.1993 (M. Koponen); Ta: Heinolan mlk., 5.6.1983, Janakkala, 12.6.1983 (M. Koponen); Sa: Mikkelin mlk., 30.7.1990, Ristiina, 14.8.1976 (M. Koponen); Tb: Keuruu, 1928 (W. Hellén), Rautalampi, 19.7.1983 (M. Koponen); Kb: Liperi, 3.7.1994 (M. Koponen).

\section{Opius (Agnopius) heringi Fischer}

Opius heringi Fischer, 1962d: 82, ơ; 1971a: 72. Opius (Misophthora) heringi; Fischer 1972: 229 (key), 245 (redescription).

Opius (Agnopius) heringi; Fischer 1982: 23. Opius (Allotypus) heringi; Tobias 1986: 58 (key, distribution).

Distribution. Austria, Finland (map 48), Germany, Ireland; Mongolia.

Host. Diptera, Agromyzidae: Phytomyza lonicerae Robineau-Desvoidy on Lonicera xylosteum, Phytomyza tenella Hering on Pedicularis palustris, Phytomyza aprilina Goureau = lonicerella (Hendel) on Lonicera periclymenium.

Material examined. $3 \sigma^{*} 3$ f from Finland (DAZH), N: Nurmijärvi, 16.6.1985, 17.7., 3.8.1994; Ta: Hattula, 1.8.1992; Sa: Kerimäki, 24.7.1982, Ristiina, 22.7.1994 (all M. Koponen leg.).

\section{Opius (Agnopius) rex Fischer}

Opius rex Fischer, 1958d: 255, ๆ; Hellén 1959: 22; Fischer 1959f: 423 (distribution, ơ); 1971a: 104.

Opius (Misophthora) rex; Fischer 1972: 228 (key),
262 (redescription); Papp 1980b: 210.

Opius (Agnopius) rex; Fischer 1982: 22, 27.

Opius (Allotypus) rex; Tobias 1986: 44.

Distribution. Austria, Crete, former Czechoslovakia, Denmark, England, Finland (map 49), France, Germany, Romania, Russia, Sweden, former Yugoslavia, Mongolia, North Korea.

Hosts. Diptera, Agromyzidae: Agromyza albipennis Meigen on Phalaris arundinacea and Arrhenatherum elatius (L.), Agromyza lucida Hendel on Deschampsia caespitosa, Agromyza nigripes Meigen on Agrostis canina, Agromyza phragmitidis Hendel on Phragmites australis, Agromyza sp. on Elymus repens, Agromyzidae gen. sp. on Calamagrostis canescens, Cerodontha (Poemyza) incisa (Meigen) on Agropyron repens (L.) and Calamagrostis epigeios (L.) Roth, Cerodontha (Poemyza) ?incisa (Meigen) on Deschampsia caespitosa, Cerodontha (Poemyza) pygmaea (Meigen) on Deschampsia caespitosa and Festuca sylvatica, Cerodontha (Poemyza) sp. on Deschampsia caespitosa, Cerodontha (Poemyza) lapplandica $($ Rydén) $=$ tatrica Nowakowski on Calamagrostis arundinacea, Dizygomyza sp. on Deschampsia caespitosa, Liriomyza flaveola (Fallén) on Festuca gigantea (L.) Vill., Liriomyza ?pusilla (Meigen) on Hieracium umbellatum, Ophiomyia sp. near pulicaria (Meigen) on Picris hieracioides, Paraphytomyza heringi (Hendel) on Fraxinus excelsior, Phytomyza astrantiae Hendel on Astrantia major, Phytomyza campanulae Hendel on Phyteuma orbiculare, Phytomyza heracleana Hering on Heracleum sphondylium, Phytomyza homogyneae Hendel on Homogyne alpina, Phytomyza hoppi Hering on Aster bellidiastrum, Phytomyza milii Kaltenbach on Deschampsia caespitosa, Poa alpina and Sesleria tatrae, Phytomyza obscurella Fallén on Aegopodium podagraria, Phytomyza senecionis Kaltenbach on Senecio nemorensis fuchsii, Phytomyza sonchi RobineauDesvoidy, Phytomyza sp. on Petasites albus, Phytomyza tussilaginis Hendel.

Material examined. $6 \sigma^{\circ} 19$ from Finland (DAZH, MZH), Ab: Lohjan kunta, 19.6.1992, Nauvo, Seili, 18.7.1978 (M. Koponen), Vihti (R. Frey); N: Helsinki, 14.8.1975, Nurmijärvi, 16.5.1979, 28.6.1980, 22.7.1987 (M. Koponen), Sibbo, Nevas, 29.6.-13.7.1986 (A. Albrecht), Siuntio, 20.8.1978 (M. Koponen); Ka: Miehikkälä, 19.6.1979 (M. Koponen); Ta: Korpilahti, 
19.8.1986, Lahti, 15.8.1980, Lammi, 27.8.1984 (M. Koponen), Pälkäne, 16.7.1976 (A.-J. Syrjänen): Sa: Mikkelin mlk., 7.7.1978, Ristiina, 23.6.1979 (M. Koponen); Tb: Keuruu, 22.7.1928 (W. Hellén), Vesanto, 28.6.1991 (M. Koponen); Kb: Liperi, 3.7.1994, Polvijärvi, 5.7.1994 (M. Koponen); Li: Inari, Ivalo, 25.6.1937 (W. Hellén leg.).

\section{Opius (Agnopius) similis Szépligeti}

Opius similis Szépligeti, 1898: 392, 405, ㅇ; Fischer 1958d: 257; 1962a: 60 (o new); 1963: 12 (new syn.).

Opius (Misophthora) similis; Fischer 1972: 229 (key), 267 (redescription).

Opius (Agnopius) similis; Fischer 1982: 22.

Opius (Allotypus) similis; Tobias 1986: 59.

Opius similiformis Fischer, 1957b: 224, ơ . Synonymised by Fischer 1963a: 12

Distribution. Austria, England, Estonia, Finland (map 50), France, Germany, Greece, Hungary, Poland, Russia, Sweden, Turkey, former Yugoslavia, Kazakhstan.

A new species to Estonia, Finland and Greece.

Hosts. Diptera, Agromyzidae: Agromyza anthracina Meigen, Agromyza genistae Hendel, Agromyza spiraeae Kaltenbach on Filipendula ulmaria, Agromyzidae gen. sp. on Calamagrostis sp., Cerodontha (Dizygomyza) caricicola (Hering) = soenderupi (Hering) on Carex hudsonii, Cerodontha silvatica Groschke, Cerodonta (Dizygomyza) bimaculata (Meigen) on Luzula pilosa, Cerodonta (Dizygomyza) chaixiana Groschke, Cerodonta (Dizygomyza) morosa (Meigen), Liriomyza amoena (Meigen) on Sambucus nigra, Liriomyza centaureae Hering on Centaurea nigra, Liriomyza pascuorum (Meigen), Metopomyza violiphaga (Hendel), Ophiomyia maura (Meigen) on Solidago virgaurea, Paraphytomomyza buhri (de Meijere) on Galium mollugo, Phytomyza alpina Groschke on Senecio jacobaea L., Phytomyza angelicae Kaltenbach on Angelica sylvestris, Phytomyza aprilina Goureau = lonicerella (Hendel), Phytomyza astrantiae Hendel on Astrantia major, Phytomyza atricornis Meigen on Centaurea jacea, Phytomyza bellidina Hering on Bellis silvestris and Bellis sp., Phytomyza campanulae Hendel on Campanula scheuchzeri and C. rotundifolia, Phytomyza lappae Goureau = lap- pina Robineau-Desvoidy on Arctium sp., Phytomyza marginella Fallén = sonchi Robineau-Desvoidy on Hieracium sp. sabaudum-group, Phytomyza obscurella Fallén on Aegopodium podagraria, Phytomyza ramosa Hendel on Knautia arvensis, Phytomyza sedicola Hering on Sedum maximum, Phytomyza solidaginis Hendel on Solidago virgaurea, Phytomyza tanaceti Hendel on Chrysanthemum cinerarifolium, Phytomyza tussilaginis Hendel on Tussilago farfara L., Phytomyza periclymenii de Meijere and Phytomyza lonicerae Robineau-Desvoidy $=x y l o s t e i$ Kaltenbach on Lonicera periclymenium, Phytomyza lonicerae Robineau-Desvoidy on Lonicera orientalis and Symphoricarpus racemosus, Amauromyza (Trilobomyza) labiatarum (Hendel) on Ballota nigra. In Finland, reared from Phytomyza virgaureae Hering by L. Tiensuu.

Material examined. $130^{*} 11$ ㅇ from Finland (DAZH, MZH), N: Helsinki, 24.8.1976, Nurmijärvi, 23.8.1980, 30.6., 12.7.1987, 11.6.1988, 22.6.1989, 14.7.1991, 28.7.1994 (M. Koponen), Sibbo, Nevas, 8.-15.7.1985 (A. Albrecht); Ka: Miehikkälä, 19.6.1979 (M. Koponen); St: Huittinen, 29.5.1993, Yläne, 14.6.1982 (M. Koponen), Ta: Hattula, 1953 (L.Tiensuu); Sa: Hirvensalmi, 6.8.1978, Kerimäki, 21.7.1982, Mikkelin mlk., 3.8.1983, 18.7.1987 (M. Koponen); Kb: Liperi, 4.8.1993, Tohmajärvi, 21.7.1982 (M. Koponen); Om: Vimpeli, 5.7.1985 (M. Koponen); Ks: Kuusamo , 3.7.1979 (M. Koponen leg.); 10 from Estonia (DAZH), Läänemaa: Puhtu, 29.5.1990 (M. Koponen); 1 ㅇ from Greece (DAZH), Corfu: Acharavi, 28.4.1994 (M. Koponen).

\section{Opius (Agnopius) similoides Fischer}

Opius similoides Fischer, 1962c: 102, 9; 1964c: 433; 1971a: 111.

Opius (Misophthora) similoides; Fischer 1972: 229 (key), 269 (redescription).

Opius (Agnopius) similoides; Fischer 1982: 22.

Opius (Allotypus) similoides; Tobias 1986: 58.

Distribution. Austria, Finland (map 51), France, Hungary, Italy, Poland, Russia.

A new species to Finland.

Host. Unknown.

Material examined. 18 f from Finland (DAZH, MZH), N: Nurmijärvi, 2.9.1979, 18.6., 28.7.1994 (M. Koponen), Pernå = Pernaja, 
23.7.1948 (̊. Nordström); St: Reposaari, 24.8.1950 (V. Lauro); Ta: Loppi, 29.7.1994 (M. Koponen); Sa: Mikkelin mlk., 12.8.1987, Punkaharju, 17.7.1982, Ristiina, 25.7.1985 (M. Koponen); Kl: Parikkala, 27.6.1990 (M. Koponen); Kb: Kitee, 22.7.1982, Polvijärvi, 5.7.1994 (M. Koponen); Om: Vimpeli, 7.7.1985 (M. Koponen); Ok: Puolanka, 29.6.1989 (M. Koponen); Oba: Pudasjärvi, 20.7.1980 (M. Koponen); Ks: Kuusamo, 4.7.1979 (M. Koponen leg.).

\section{Opius (Agnopius) tirolensis Fischer}

Opius tirolensis Fischer, 1958d: 258, ; 1971a: 117.

Opius (Misophthora) tirolensis; Fischer 1972: 230 (key), 275 (redescription); Papp 1979a: 90.

Opius (Agnopius) tirolensis; Fischer 1982: 23. Opius (Allotypus) tirolensis; Tobias 1986: 60.

Distribution: Austria, Denmark, Finland (map 52), Germany, Hungary, Italy, Poland, Russia (Leningrad region), former Yugoslavia.

A new species to Finland and Poland.

Host. Diptera, Agromyzidae: Phytomyza flavicornis Fallén on Urtica dioica.

Material examined. 6 ㅇ from Finland (DAZH), Ab: Lohjan kunta, 19.6.1992, Perniö, 20.9.1994 (M. Koponen); N: Nurmijärvi, 19.6.1993 (M. Koponen); Ta: Korpilahti, 20.8.1986 (M. Koponen); Sa: Mikkelin mlk., 18.7.1987 (M. Koponen); Ok: Kuhmo, 6.7.1980 (M. Koponen leg.); and 2 ㅇ from Poland (DAZH), Bialowieza, 6.7.1988 (M. Koponen leg.).

\section{Subgenus Allotypus Foerster}

Allotypus Foerster, 1862: 259. Type species: Opius irregularis Wesmael.

Subgenus Allotypus; Fischer 1972: 70 (key), 280 (descr.).

\section{Opius (Allotypus) geniculatus Thomson}

Opius geniculatus Thomson, 1895: 2179, 우 후; Hellén 1946: 132; Fischer 1958e: 214; 1971a: 69. Opius (Allotypus) geniculatus; Fischer 1972: 280 (key), 282 (redescription); Papp 1979: 79; Tobias 1986: 63.

Opius albicoxis Marshall, 1898: 236, 우 ㅈ․ Synonymised by Fischer 1958d: 214.
Distribution. Austria, former Czechoslovakia, Denmark, England, Finland (map 53), France, Germany, Hungary, Poland, Romania, Russia, Sweden, Japan.

Hosts. Diptera, Tephritidae: Trypeta immaculata (Macquart) on Taraxacum sp., Vidalia cornuta (Scopoli).

Material examined. $160^{\star}, 30$ from Finland (DAZH, MZH), Ab: Perniö, 19.9.1994 (M. Koponen); N: Helsingfors = Helsinki, 17.4.1944 (A. Nordman), 11.8., 3.9.1975, 4.-18.9.1978, 18.7.22.8., 26.9.1979, 13.9.1982, 28.8.-2.9.1980 (M. Koponen), Nurmijärvi, 23.7., 23.8.1980, 17.7., 21.8.1981, Porvoon mlk., 23.7.1979 (M. Koponen), Sibbo, Nevas, 1.-14.8.1988 (A. Albrecht), Vantaa, 31.8.1975, 24.8.1979, 21.8.1991 (M. Koponen); St: Mäntyluoto, 16.7.1950, Reposaari, 7.8.1950, 31.7.1966 (V. Lauro); Ta: Hauho, 13.8.1935 (W. Hellén), Kangasala (R. Frey), Korpilahti, 19.8.1986, Lahti, 17.7.1987, Nastola, 17.8.1980 (M. Koponen), Sääksmäki, 1930, 20.8.1934 (E. Kivirikko); Sa: Joutseno, 23.7.1937 (E. Thuneberg), Mikkelin mlk., 23.-24.8.1975, 19.8.1994, Ristiina, 6.9.1987 (M. Koponen); $10^{*}$, 1 우 from Poland (DAZH), Bialowieza, 7.7.1988 (M. Koponen leg.), and 2 ㅇ from Russia (MZH, DAZH), Karelia: Kexholm (S. Sahlberg); Moscow, 8.7.1977 (M. Koponen leg.).

\section{Opius (Allotypus) irregularis Wesmael}

Opius irregularis Wesmael, 1835: 132, $9 \sigma^{*}$; Fischer 1958e: 216; 1971a: 77; Hellén 1959: 22; Wharton 1987: 66.

Allotypus irregularis; Foerster 1862: 259.

Opius (Allotypus) irregularis; Fischer 1972: 280

(key), 284 (redescription); Tobias 1986: 60.

Opius bipustulatus Fischer, 1958e: 211, o; ;. Fischer 1962a: 47 ( 9 nov.). Synonymised by Fischer 1971a: 77.

Distribution. One of the commonest species of Opius in west, north and central Europe, including Finland (map 54), Balkans, Russia, Japan, North America.

Host. Diptera, Ephydridae: Hydrellia griseola (Fallén) on Poa trivialis.

Material examined. $14 \sigma^{\star}, 22 \%$ from Finland (DAZH, MZH), Al: Lemland, Flaka, 8.-12.6.1954 (Waselius); Ab: Runsala = Turku, Ruissalo (Ingelius), Vihti (Ingelius); N: Espoo, 4.9.1979 (M. 
Koponen), Hangö = Hanko, 1931 (W. Hellén), Helsinki, 31.8., 11.9.1978, 12.8.1979, 6.-29.6. 1980, Hyvinkää, 4.8.1979, Nurmijärvi, 2.9.1979, 14.9.1987, 26.6.1989, 10.8.1994, Porvoon mlk., 23.7.1979, 3.6.1982, Vantaa, 22.8.1978, 11.8. 1979, 6.8.1980, 21.8.1991 (M. Koponen); St: Reposaari, 29.8.1955 (V. Lauro); Ta: Jämsä, 26.8.1991 (M. Koponen), Kangasala (R. Frey); Sa: Mikkelin mlk., 22.8.1975, 19.8.1994 (M. Koponen); Obb: Simo, 17.7.1980 (M. Koponen); Ks: Kuusamo, 30.6.1979 (M. Koponen); 1 \% , 1 우 from Poland (DAZH); and 10 from Japan (DAZH), Kyoto, 20.8.1988 (M. Koponen leg.).

\section{Opius (Allotypus) saevulus Fischer}

Opius saevulus Fischer, 1958e: 217, $0^{*} ; 1971 \mathrm{a}:$ 108.

Opius (Allotypus) saevulus; Fischer 1972: 280 (key), 287 (redescription); Tobias 1986: 63.

Distribution. Austria, England, Finland (map 55), Hungary.

A new species to Finland.

Hosts. Diptera, Scatophagidae: Parallelomma (= Chylizosoma) vittatum (Meigen) on Listera ovata and Orchis mascula L., Tephritidae: Philophylla heraclei (Linnaeus) on Pastinaca sativa.

Remark. Host records need verification.

Material examined. 10 from Finland (DAZH), N: Siuntio, 30.8.1981 (M. Koponen leg.).

\section{Opius (Allotypus) saevus Haliday}

Opius saevus Haliday, 1837: 209, 우 ðే; Fischer 1958e: 218; 1967a: 344 (host); 1971a: 108.

Opius (Allotypus) saevus; Fischer 1972: 280 (key), 289 (redescription); Papp 1979: 89; 1982a: 245; Tobias 1986: 63 (key, distribution).

Distribution. Austria, Denmark, England, Finland (map 56), Hungary, Scotland, Armenia, North Korea.

A new species to Finland.

Host. Diptera, Agromyzidae: Nemorimyza posticata (Meigen).

Material examined. $4 \delta^{\star}, 69$ from Finland (DAZH, MZH), N: Kirkkonummi, 24.7.1991 (M. Koponen), Sibbo, Nevas, 21.7-18.8.1985 (A. Albrecht), Sipoo, 27.8.1975 (M. Koponen); Sa: Ristiina, 6.8.1978 (M. Koponen); Kb: Kitee, 19.7.1982, Liperi, 4.8.1993 (M. Koponen); Obb:
Simo, 17.7.1980 (M. Koponen); Ks: Kuusamo, 4.7.1979 (M. Koponen leg.).

\section{Subgenus Eutrichopsis Foerster}

Eutrichopsis Foerster, 1862: 260. Type species: Eutrichopsis munda Foerster.

Subgenus Eutrichopsis; Fischer 1986: 609 (key), 610.

\section{Opius (Eutrichopsis) mundus (Foerster)}

Eutrichopsis munda Foerster, 1862: 260.

Opius mundus; Fischer 1957a: 49, 9 ; 1971a: 89. Opius (Nosopoea) mundus; Fischer 1972: 291 (key), 320 (redescription).

Opius (Allotypus) mundus; Tobias 1986: 66.

Opius (Eutrichopsis) mundus; Fischer 1986: 610.

Distribution. Austria, former Czechoslovakia, England, Finland (map 57), Germany, Hungary, Kazakhstan.

A new species to Finland.

Host. Unknown.

Material examined. 2 ㅇ from Finland (DAZH), N: Nurmijärvi, 25.8.1987 (M. Koponen); Kl: Uukuniemi, 20.7.1983 (M. Pulkkinen leg.).

\section{Subgenus Nosopoea Foerster}

Nosopoea Foerster, 1862: 260. Type species: Opius cingulatus Wesmael.

\section{Opius (Nosopoea) altimontanus Fischer}

Opius altimontanus Fischer, 1969a: 40, 우우 1971a: 41; 1974: 82.

Opius (Nosopoea) altimontanus; Fischer 1972: 292 (key, redescription); Papp 1982a: 243; Fischer 1986: 612 (key).

Opius (Allotypus) altimontanus; Tobias 1986: 68.

Distribution. Austria, former Czechoslovakia, Finland (map 58), Hungary, Romania, Russia (Murmansk region).

A new species to Russia.

Host. Unknown.

Material examined. $13 \sigma^{*}, 7$ 우 from Finland (DAZH), Ab: Perniö, 19.9.1994; N: Helsinki, 9.8.1976, 9.-16.9.1978, Kirkkonummi, 23.24.7.1991, Nurmijärvi, 30.7.1982, 21.-30.8.1987, 18.7.1992, 8.9.1994 (M. Koponen); Ta: Juupajoki, 
15.6.1982 (Y. Zhongqi); Sa: Ristiina, 23.7.1978, 28.7.1979, Suomenniemi, 23.7.1978, Valkeala, 28.7.1977 (M. Koponen); Obb: Tornio, 6.7.1982 (M. Koponen); and $10^{7}$ from Russia (MZH), Murmansk region: Yläluostari, 1928 (W. Hellén leg.).

\section{Opius (Nosopoea) ambiguus Wesmael}

Opius ambiguus Wesmael, 1835: 131, o*; Fischer 1960: 68; 1971a: 42.

Opius (Nosopoea) ambiguus Fischer 1972: 292 (key, ㅇ), 295 (redescription); 1986: 612 (key). Opius (Allotypus) ambiguus; Tobias 1986: 68. Opius longipes Fischer, 1957b: 218, 오. Synonymised by Fischer 1971a: 42.

Opius phytomyzae Fischer, 1957b: 220, क; Hellén 1959: 22. Synonymised by Fischer 1971a: 42. Opius reptantis Fischer, 1957b: 223, ๆ; Hellén 1959: 22. Synonymised by Fischer 1971a: 42.

Distribution. Austria, Denmark, England, Finland (map 59), France, Germany, Ireland, Poland, Russia, Sweden, Switzerland, Mongolia.

Hosts. Diptera, Agromyzidae: Agromyza albipennis Meigen on Phalaris arundinacea, Agromyza anthracina Meigen on Urtica dioica, Agromyza nigrescens Hendel, Agromyza reptans Fallén, Agromyza sp. on Trifolium sp., Agromyza pseudoreptans Nowakowski $=$ urticae Nowakowski, preocc. on Urtica dioica, Cerodontha (Phytagromyza) flavocingulata (Strobl) on Festuca gigantea, Cerodontha (Poemyza) incisa (Meigen) on Calamagrostis arundinacea, Cerodontha (Poemyza) luzulae Groschke on Luzula sylvatica, Cerodonta (Dizygomyza) caricicola (Hering) $=$ soenderupi $($ Hering) on Carex pendula, Liriomyza pusio (Meigen) = graminicola de Meijere on Arrhenatherum elatius, Nemorimyza posticata (Meigen) on Solidago virgaurea, Paraphytomyza buhri (de Meijere) on Galium album = mollugo, Phytomyza angelicae Kaltenbach = aegopodii Hendel on Aegopodium podagraria and Angelica sylvestris, Phytomyza alpina Goureau on Senecio jacobaea, Phytomyza angelicastri Hering, Phytomyza lappae Goureau = lappina Robineau-Desvoidy on Arctium sp., Phytomyza marginella Fallén = sonchi Robineau-Desvoidy on Hieracium sp. sabaudum group, Phytomyza milii Kaltenbach on Deschampsia caespitosa, Phytomyza montana Groschke, Phytomyza sphondyliivora Spencer on Heracleum sphondylium.

Material examined. $90^{\circ}, 3+$ from Finland (DAZH, MZH), N: Hertonäs = Helsinki, Herttoniemi, 10.8.1908 (L. von Essen), Sibbo, Nevas, 22.-28.6., 12.-18.7.1986 (A. Albrecht), Tenala, Harpar Lillträsk, 28.7.-3.8.1988 (A. Albrecht \& K. Keynäs); St: Reposaari, 1.7.1951 (V. Lauro); Sa: Ristiina, 28.7.1986 (M. Koponen); Kl: Parikkala, 18.6.1945 (W. Hellén leg.).

\section{Opius (Nosopoea) celsus Haliday}

Opius celsus Haliday, 1837: 209, ơ ; Fischer 1959g: 66; 1971a: 52.

Opius (Nosopoea) celsus; Fischer 1972: 292 (key), 297 (redescription, ㅇ); 1986: 612 (key).

Opius (Allotypus) celsus; Tobias 1986: 68 (key, distribution).

Distribution. Austria, Denmark, England, Estonia, Finland (map 60), France, Germany, Ireland, Sweden, Switzerland, Ukraine, China.

A new species to Finland and Estonia.

Host. Diptera, Agromyzidae: Agromyza albipennis Meigen on Phalaris arundinacea.

Material examined. $20^{\circ}, 2$ from Finland (DAZH, MZH), N: Sibbo, Nevas, 7.-15.7.1985 (A. Albrecht); Ok: Kajaani, 5.7.1991 (M. Koponen); and 1 o from Estonia (DAZH), Läänemaa: Vatla, 3.6.1990 (M. Koponen leg.).

\section{Opius (Nosopoea) cingulatus Wesmael}

Opius cingulatus Wesmael, 1835: 120, 우 후; Fischer 1959g: 67; Hellén 1959: 21; Fischer 1967b: 141; 1971a: 54; Wharton 1987: 65.

Nosopoea cingulatus; Foerster 1862: 216.

Opius (Nosopaeus) cingulatus; Thomson 1895: 2191, 우 $0^{\star}$.

Opius (Nosopoea) cingulatus; Fischer 1972: 292 (key), 303 (redescription); 1986: 612 (key); Papp 1978b: 368; 1982c: 184.

Opius (Allotypus) cingulatus; Tobias 1986: 70.

Opius (Nosopaeus) stramineipes Thomson, 1895: 2192, 우 $0^{\star}$. Synonymised by Fischer 1971a: 54 .

Nosopoeus stramineipes; Krogerus 1927: 47: 54. Opius (Nosopaeus) dentifer Thomson, 1895: 2192, ㅇ. Synonymised by Fischer 1971a: 54.

Distribution. Austria, Denmark, Estonia, Finland (map 61), Germany, Great Britain, Greece, Italy, Poland, Russia (Leningrad region, Krogerus 
1927), Sweden, Armenia.

Hosts. Diptera, Agromyzidae: Agromyza albitarsis Meigen on Populus tremula, Agromyza nigrella Rondani on Triticum sp., Agromyza sp. on Bromus asper, Agromyza potentillae (Kaltenbach) $=$ spiraeae $($ Kaltenbach) on Filipendula ulmaria, Agromyza? pseudoreptans Nowakowski = urticae Nowakowski, preocc. on Urtica dioica, Ophiomyia maura (Meigen), Napomyza ?evanescens (Hendel) on Ranunculus lanuginosus, Liriomyza morio (Brischke) on Asperula odorata and Galium sylvaticum, Amauromyza (Trilobomyza) labiatarum (Hendel) on Ballota nigra; Tephritidae: Philophylla heraclei (Linnaeus).

Material examined. $320^{\star}, 32$ o from Finland (DAZH, MZH), Al: Saltvik (R. Frey); Ab: Nauvo, Seili, 20.7.1978, Perniö, 19.9.1994 (M. Koponen), Nystad = Uusikaupunki, 1920, 1921, 14.7.1923 (W. Hellén), Vichtis = Vihti (R. Frey); N: Elimäki, 16.6.1984 (M. Koponen), Esbo = Espoo, 10.-17.7.1910, Hangö = Hanko, 12.7.1914, 1931 (W. Hellén), 9.-14.8.1990 (A. Albrecht), H:fors = Helsinki (R. Frey), 15.8., 1.9.9.1978, 6.9.1980, 15.6., 2.9.1981 (M. Koponen), 12.9.1982 (Y. Zhongqi), Nurmijärvi, 3.9.1977, 23.8.1980, 14.7.1991 (M. Koponen), Sibbo, Kitö (Stenius), Sibbo, Nevas, 7.7.-18.8.1985, 7.7.17.8.1986, 13.-20.6.1988 (A. Albrecht), Sipoo, 13.8.1977, Siuntio, 20.8.1977, Tuusula, 22.8.1976, Vantaa, 31.8.1975, 16.8.1978 (M. Koponen); St: Reposaari, 4.-24.7.1950, 9.7.1951, 16.8.1954 (V. Lauro); Ta: Lammi, 8.7.1975, 12.7., 4.9.1976 (M. Koponen); Sa: Anttola, 15.8.1976 (M. Koponen), Imatra, 16.5.1954 (O. Nybom), Mikkelin mlk., 22.8.1975, 4.7.1978, 15.9.1979, 5.7.1980, 23.6.1983, 4.8.1989, Ristiina, 14.8.1976, 25.6.1983 (M. Koponen); Kl: Parikkala, 27.6.1990 (M. Koponen); Tb: Keuruu, 14.7.1928 (W. Hellén), Konnevesi, 19.8.1986 (M. Koponen); Ok: Kajaani, 5.7.1991 (M. Koponen); 10 from Estonia (DAZH), Saaremaa: Viidumägi, Suurmägi, 1.6.1990 (M. Koponen); $10^{7}$ from Russia (MZH), Leningrad region: Terijoki, Kellomäki, 31.8.1927 (W. Hellén); and 1 i from Poland (DAZH), Bialowieza, 6.7.1988 (M. Koponen leg.).

\section{Opius (Nosopoea) circulator (Nees)}

Bracon circulator Nees, 1834: 54, 우 ه*. Opius circulator; Wesmael 1835: 131; Fischer 1959g: 69; 1971a: 54; Hellén 1959: 21.

Opius (Nosopoea) circulator; Fischer 1972: 292

(key), 305 (redescription); Papp 1981c: 139, 146; Fischer 1984a: 129; 1986: 611.

Opius (Allotypus) circulator; Tobias 1986: 66.

Opius (Nosopoea) eversus Papp, 1981c: 140, 146, . Synonymised by Fischer 1984a: 131.

Distribution. Austria, Finland (map 62), France, Germany, Poland, Russia, Sweden, North Korea.

A new species to Poland and Sweden.

Host. Unknown.

Material examined. 30*, 52 f from Finland (DAZH, MZH), Ab: Nauvo, Seili, 17.7.1978 (M. Koponen), Vichtis = Vihti (R. Frey), 9.8.1981 (M. Koponen); N: Helsinki, 4.-19.8.1976, 20.7., 12.8.1977, Kirkkonummi, 23.7.1991, Nurmijärvi, 16.8.1978, 5.8.1979, 28.8.1982, 5.9.1984, 28.8.1987, 5.9.1989, 18.7.1992, 9.8.1994, Porvoon mlk., 10.9.1980, Sipoo, 27.8.1975, 26.7.1981 (M. Koponen); Ka: Hamina, 30.7.1953 (E. Thuneberg), Kotka, 31.7.1977 (M. Koponen); Ta: Hausjärvi, 20.8.1981, Heinolan mlk., 21.8.1983, Tammela, 1.7.1978 (M. Koponen); Sa: Mikkelin mlk., 28.7.1990, Punkaharju, 17.7.1982, Ristiina, 14.8.1976, 30.8.1985, 29.7.1986, Suomenniemi, 23.7.1978, Valkeala, 28.7.1977 (M. Koponen); Tb: Rautalampi, 17.-18.7.1983 (M. Koponen); Kb: Kitee, 22.7.1982, Liperi, 4.8.1993, Tohmajärvi, 20.7.1982 (M. Koponen); Obb: Simo, 19.7.1980 (M. Koponen); Li: Inari, 30.7.1993 (M. Koponen); 1 ㅇ from Poland (DAZH), BialowieZa, 9.7.1988; and 1 ㅇ from Sweden (DAZH), Vb: Brännland, 14.7.1980 (M. Koponen leg.).

\section{Opius (Nosopoea) maculipes Wesmael}

Opius maculipes Wesmael 1835: 128, o o ; Fischer 1959g: 72; 1971a: 84; Perepetcheyenko 1994: 37 (distribution).

Opius (Nosopoea) maculipes; Fischer 1972: 291 (key), 317 (redescription); 1986: 611; Papp 1978a: 142; 1982c: 184.

Opius (Allotypus) maculipes; Tobias 1986: 66 (key).

Opius addendus Fischer, 1959h: 20, ๆ; 1962b: 65, $\sigma^{*}$. Synonymised by Fischer 1971a: 84.

Opius turcmenicus Fischer 1959c: 28, ․ Synonymised by Fischer 1971a.

Distribution. Austria, Belgium, the Canary Is- 
lands, former Czechoslovakia, Denmark, England, Finland (map 63), Germany, Greece, Hungary, Ireland, Moldova, Sweden, Switzerland, Ukraine, Tunisia, Turkmenistan.

A new species to the Canary Islands and Finland.

Hosts. Diptera, Agromyzidae: Agromyza ambigua Fallén = niveipennis Zetterstedt on Secale cereale, Agromyza bicophaga Hering on Vicia tenuifolia, Agromyza lathyri Hendel on Lathyrus latifolius, Agromyza nigrella Rondani on Triticum sp., Agromyza nigriceps Hendel on Humulus lupulus, Agromyza potentillae (Kaltenbach) = spiraeae (Kaltenbach) on Filipendula ulmaria.

Material examined. 4 ㅇ from Finland (DAZH), N: Nurmijärvi, 16.5.1979 (M. Koponen); Ta: Kuhmoinen, 4.6.1920 (Y. Hukkinen), Lammi, 14.7.1976; Sa: Mikkelin mlk., 11.7.1981 (M. Koponen); 1 i from Greece (DAZH), Corfu: Acharavi, 24.4.1994 (M. Koponen); and 5 o from the Canary Islands (DAZH), Tenerife: Sta Ursula, La Quinta, 8.-9.12.1993 (M. Koponen leg.).

\section{Opius (Nosopoea) pirchitticola Fischer}

Opius (Nosopoea) pirchitticola Fischer, 1974: 83; 1986: 611.

Opius (Allotypus) pirchitticola; Tobias 1986: 66.

Distribution. Austria, Finland (map 64).

A new species to Finland.

Host. Unknown.

Material examined. 2G, 1E from Finland (DAZH), Li: Inari 2.7., 6.7.1989 (M. Koponen leg.).

\section{Opius (Nosopoea) seebensteinensis Fischer}

Opius seebensteinensis Fischer, 1959g: 63, G. Opius (Nosopoea) seebensteinensis; Fischer 1972: 292 (key), 323 (redescription); 1986: 612 (key). Opius (Allotypus) seebensteinensis; Tobias 1986: 70.

Distribution. Austria, Finland (map 65), Germany, Hungary, Italy.

A new species to Finland.

Hosts. Diptera, Agromyzidae: Phytomyza bellidina Hering on Bellis sp., Phytomyza ranunculi Schrank on Ranunculus repens.

Material examined. $20^{7}$ from Finland (DAZH), N: Helsinki, 20.7.1977; Sb: Kuopio, 27.6.1989
(M. Koponen leg.).

\section{Opius (Nosopoea) sigmodus Papp}

Opius sigmodus Papp, 1980: 43, 46; 1981b: 270, 우.

Opius (Nosopoea) sigmodus; Fischer 1986: 612 (key), 652 (redescription).

Opius (Allotypus) sigmodus; Tobias 1986: 70.

Distribution: Finland (map 66), Hungary.

A new species to Finland.

Host. Unknown.

Material examined: 10 from Finland (MZH), Al: Mariehamn, 7.1946, ad lucem(W. Hellén leg.).

\section{Opius (Nosopoea) speciosus Fischer}

Opius speciosus Fischer, 1959g: 74, 욱 1971a: 112; 1974: 84.

Opius (Nosopoea) speciosus; Fischer 1972: 291

(key), 324 (redescription); 1986: 611 (key).

Opius (Allotypus) speciosus; Tobias 1986: 63, 66 (key).

Distribution. Armenia, Austria, Finland (map

67), France, Hungary, Russia.

A new species to Finland.

Host. Unknown.

Material examined: 2E from Finland (DAZH), N: Helsinki, 28.7.1980 (M. Koponen leg.).

Opius (Nosopoea) tuberculiferFischer ssp. tuberculifer Fischer

Opius tuberculifer Fischer, 1958f: 179, E; 1971a: 119.

Opius (Nosopoea) tuberculifer, Fischer 1972: 291 (key), 328 (redescription); 1986: 612 (key).

Opius (Allotypus) tuberculifer; Tobias 1986: 66.

Distribution. Austria, Finland (map 68), Germany, Hungary, Switzerland.

A new species to Finland.

Host. Unknown.

Material examined. 9 을 from Finland (DAZH), St: Yläne, 17.6.1982; Oba: Ii, 20.7.1980 (M. Koponen leg.).

Opius (Nosopoea) tuberculifer Fischer ssp. diabolicus Fischer

Opius diabolicus Fischer, 1961: 8, ๆ; 1969a: 47 
(distribution, ơ nov.); 1971a: 61; 1974: 82.

Opius (Nosopoea) diabolicus; Fischer 1972: 292

(key), 312 (redescription); 1986: 612 (key).

Opius (Allotypus) diabolicus; Tobias 1986: 66.

Opius (Nosopoea) tuberculifer Fischer ssp. diabolicus; Fischer 1986: 612 (nov. stat. of diabolicus).

Distribution. Austria, Finland (map 69), Switzerland.

A new subspecies to Finland.

Host. Unknown.

Material examined. $10^{*}, 12$ from Finland (DAZH, MZH), N: Nurmijärvi, 5.8.1979 (M. Koponen), Sibbo, Nevas, 4.-31.8.1985, 1.14.8.1988 (A. Albrecht); St: Eura, 7.7.1981 (M. Koponen); Ok: Kuhmo, 10.7.1980 (M. Koponen); Obb: Ranua, 19.7.1980 (M. Koponen leg.).

\section{Opius (Nosopoea) viennensis Fischer}

Opius viennensis Fischer, 1959g: 76, o o o ; 1971a: 121.

Opius (Nosopoea) viennensis; Fischer 1972: 292 (key), 329 (redescription); 1986: 612 (key); Papp 1985: 204.

Opius (Allotypus) viennensis; Tobias 1986: 68. Distribution: Austria, former Czechoslovakia, Finland (map 70), France, Hungary.

A new species to Finland.

Host. Unknown.

Material examined: 1 우 from Finland (MZH), Ta: Pälkäne, 23.6.1921 (M. Hellén); and $10^{\pi}$ from France (MZH), Annecy (W. Hellén leg.). 


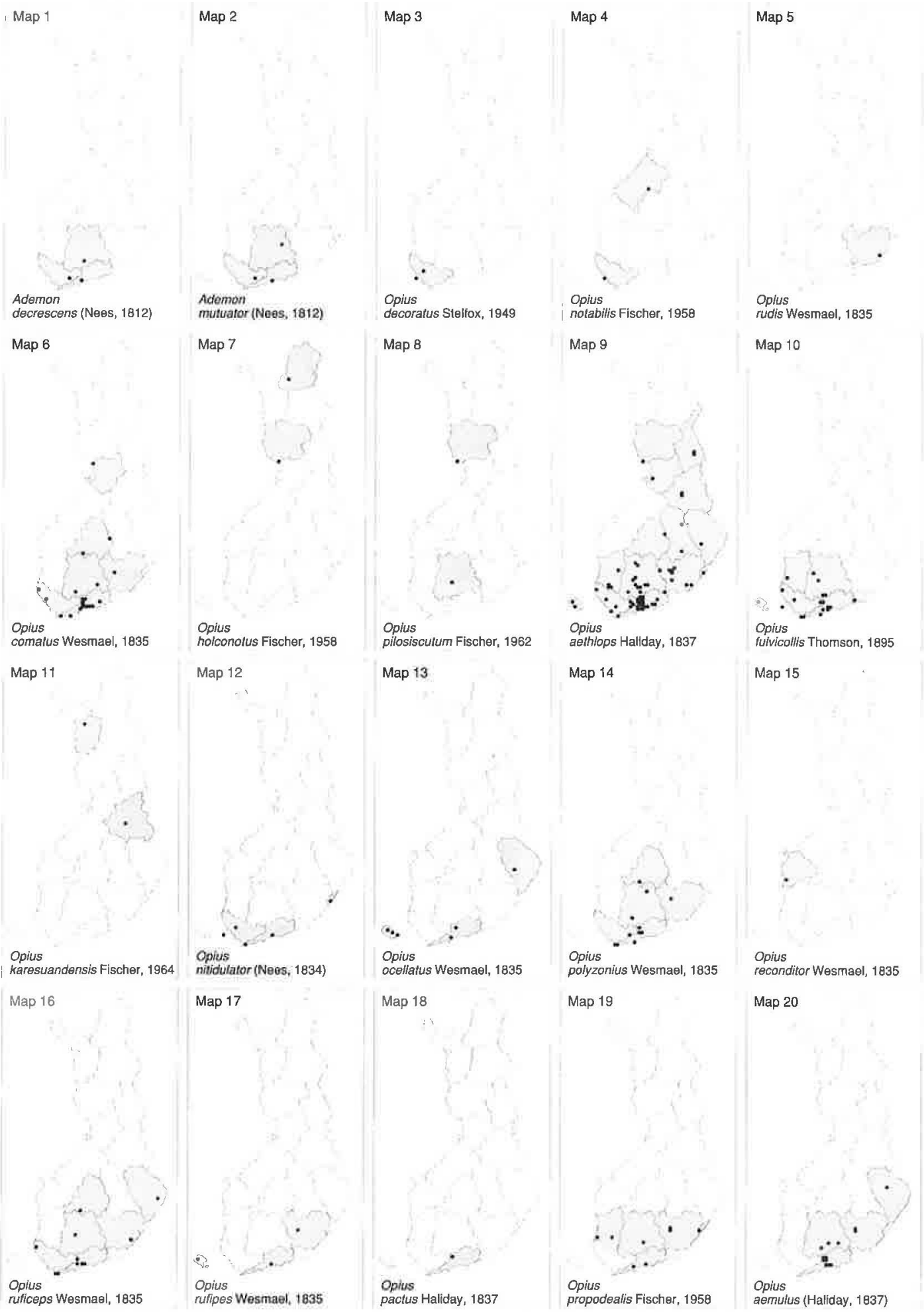



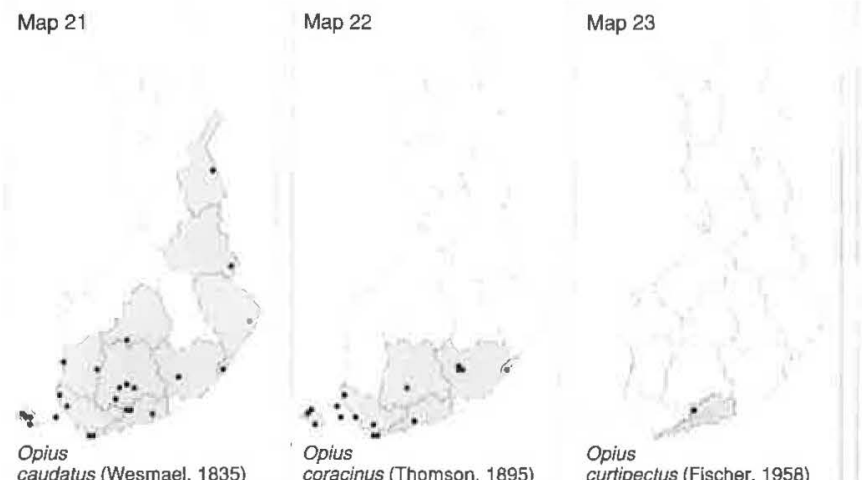

Map 24

Map 25
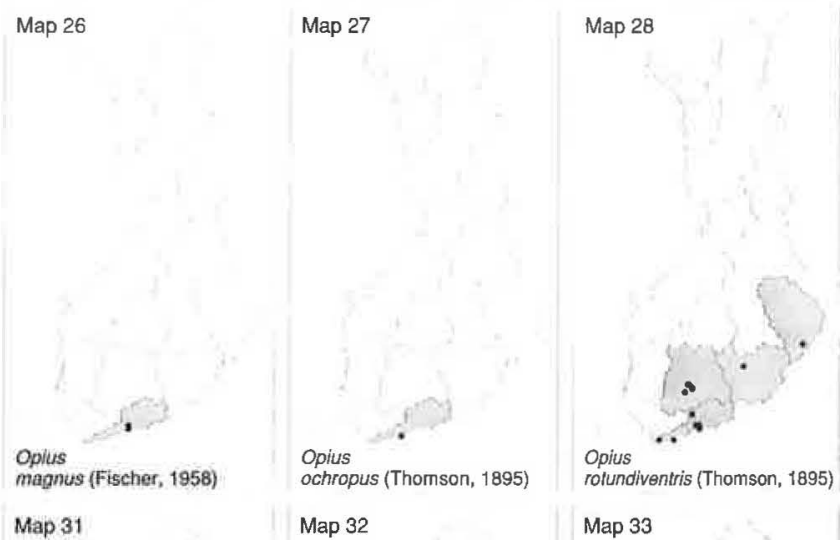

Opius

fasciatus (Thomson, 1895)

Map 29

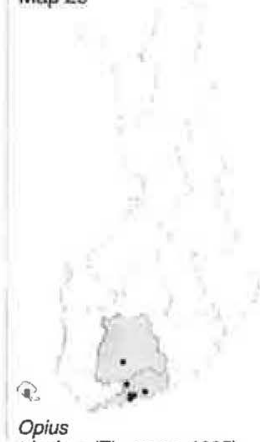

Map 33

Opius

trisulcus (Thomson, 1895)

Map 34

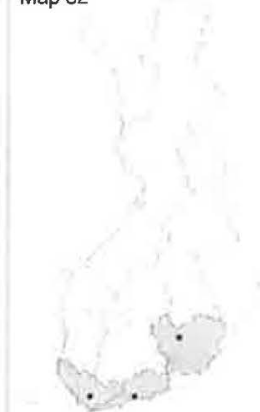

Opius

aureliae Fischer, 1957

zelotes (Marshall, 1891)

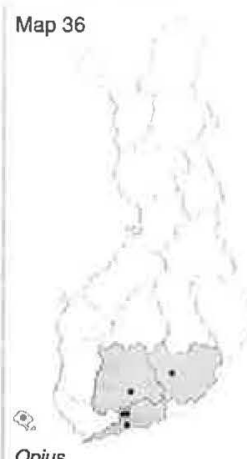

Opius

curvatus Flscher, 1957
Map 37

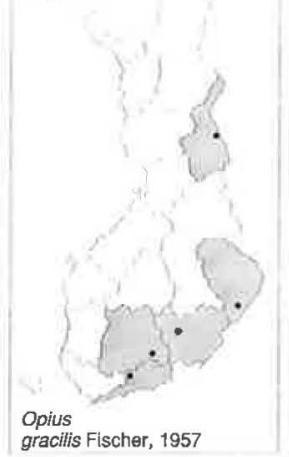

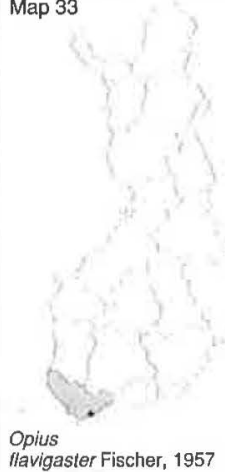

Map 38

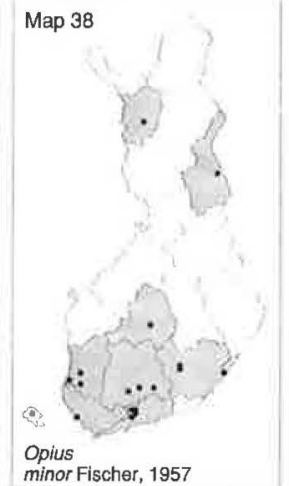

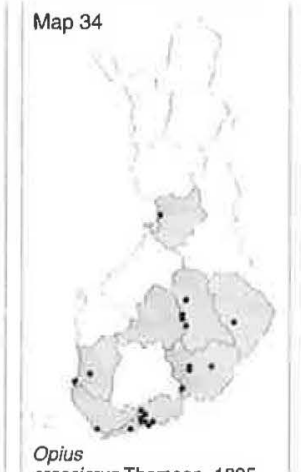

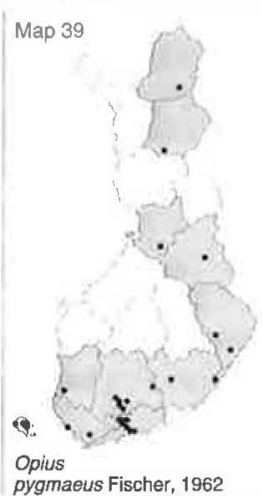

$\Rightarrow \quad \dot{x}$

Opius

hilaris (Fischer, 1962)

Map 30

truncatus (Wesmael, 1835)

Map 35

Opius

Ischeki Fischer, 1957

Map 40

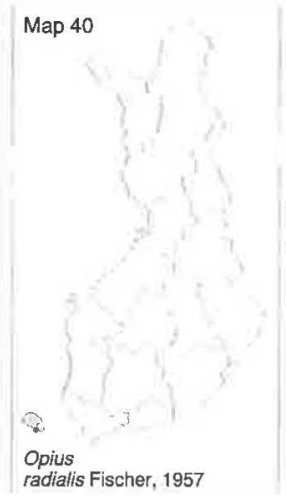




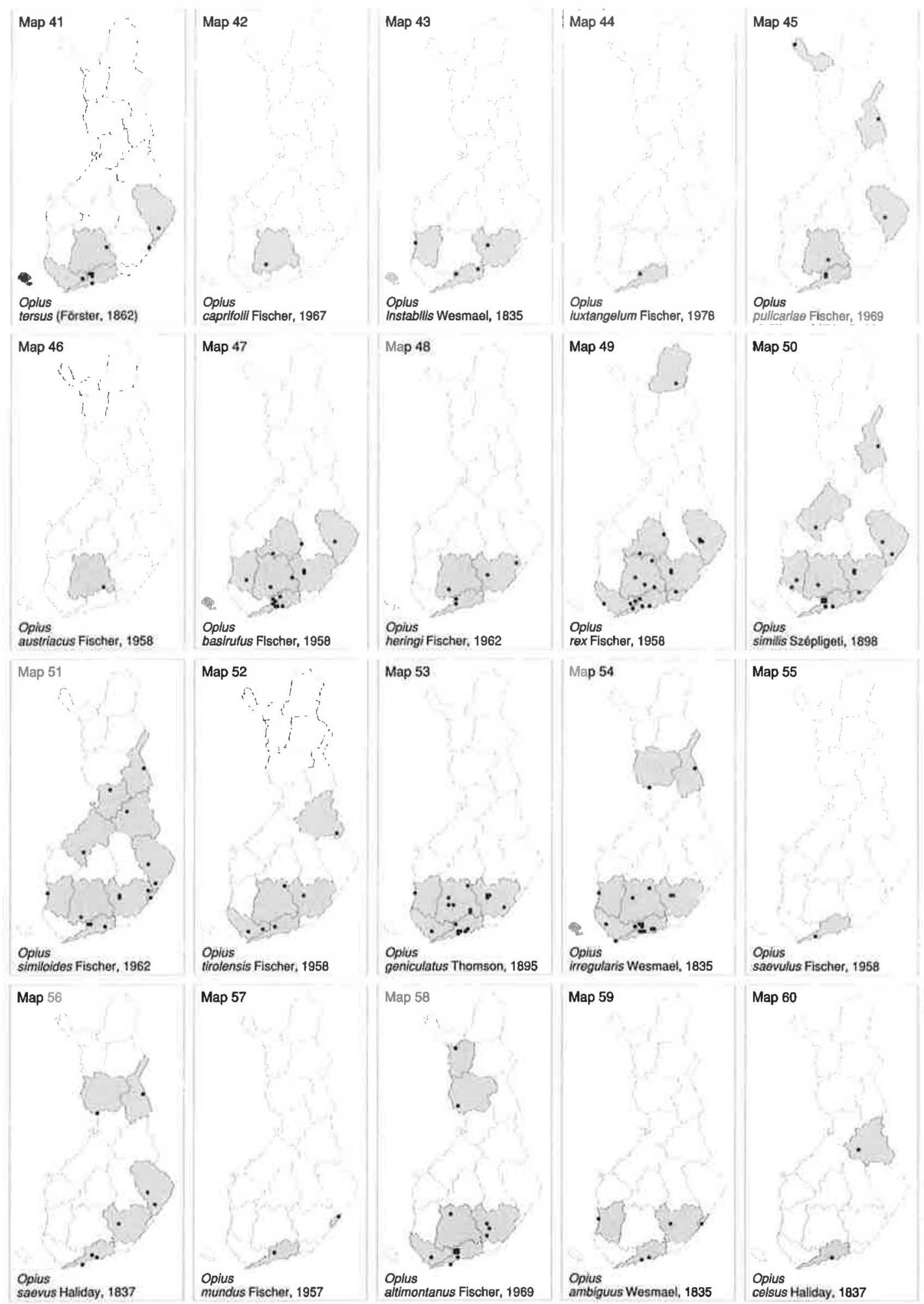




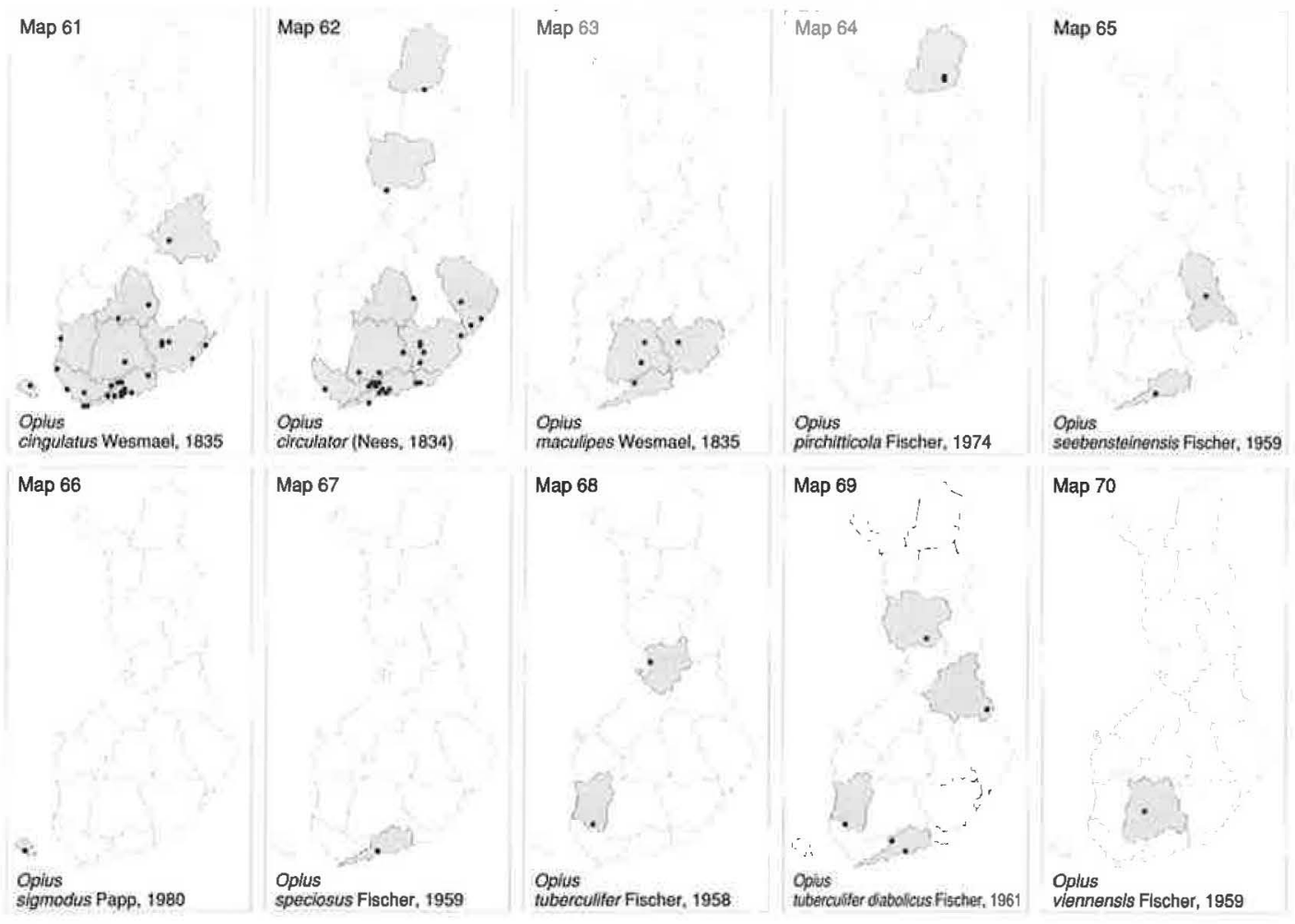

